\title{
General trajectory surface hopping method for ultrafast nonadiabatic dynamics
}

Sebastian Mai, ${ }^{a}$ Felix Plasser, ${ }^{a}$ Philipp Marquetand, ${ }^{a}$ and Leticia González ${ }^{a *}$

${ }^{a}$ Institute of Theoretical Chemistry, Faculty of Chemistry, University of Vienna, Währinger Str. 17, 1090 Vienna, Austria.

Corresponding contributor. E-mail: leticia.gonzalez@univie.ac.at

Theoretical and Computational Chemistry Series No. 13

Attosecond Molecular Dynamics

Edited by Marc J. J. Vrakking and Franck Lepine DOI: 10.1039/9781788012669-00348 


\begin{abstract}
Nonadiabatic dynamics is a central concept in the study of ultrafast chemical and physical processes, especially light-induced processes. One method to computationally study such processes is provided by the "Surface Hopping including ARbitrary Couplings" (SHARC) approach, which we present here. We focus on a general description of the involved approximations, and the strengths and limitations of the approach. We also discuss the choice of electronic structure method and give an overview over techniques to analyze the trajectories obtained in the simulations.
\end{abstract}




\subsection{Introduction}

Chemistry can be viewed as the motion of nuclei and electrons. Thus, one approach to understand chemistry is to follow that motion. Such an endeavor is highly complex due to the typical presence of non-Born-Oppenheimer (nonadiabatic) couplings and spin-orbit couplings (SOCs), among other factors. The motion of nuclei and electrons and their accompanying interactions occur already on the time scale of femto- and attoseconds $\left(10^{-15}\right.$ and $10^{-18}$ seconds), respectively. The investigation of these short time scales is only possible with light, and considerable effort is being devoted to create ever shorter laser pulses in order to observe chemical processes in real time. ${ }^{1-6}$

With the advent of time-resolved spectroscopy, ${ }^{7-12}$ photochemical and photophysical processes can be studied using pump-probe experiments. In a pump-probe experiment, the first pulse (pump) excites the system, starting the reaction in an electronic excited state. The course of the reaction is then interrogated by a second pulse (probe), by repeating the experiment with different time delays between the two pulses and thus providing a timedependent picture of the chemical reaction. The actual observable in these experiments depends on the characteristics of the laser pulses: If a good time resolution is aimed at, it is desirable to employ short pulses, which then necessarily feature large energetic bandwidthsas for example in the case of studying high-harmonic generation or ionization processes. Both the signals from ionization and high harmonic generation are able to trace nuclear and electronic motion. The reason is that the ionization probability and also its counterpart, the recombination probability in high harmonic generation, depend on the nuclear and electronic configuration of the system. ${ }^{13}$ In this sense, two types of attosecond pump-probe experiments can be conceived: (i) a standard (femtosecond) pump pulse excites a molecule and probing is executed afterwards by generating high harmonics within the excited molecule, ${ }^{13}$ or (ii) high harmonics are generated externally, and only the attosecond pulses from the high harmonics generation are used to investigate the molecule of interest. ${ }^{14}$ In either case, the initial excitation leads to nuclear and electronic motion, which in most cases implies that excited electronic states are populated. As the accompanying dynamics of the molecule can be quite complex, theory plays an essential role in deciphering the molecular changes which are encoded in the experimental signals. ${ }^{15}$

In this chapter, we discuss our on-going efforts in investigating ultrafast excited-state processes using the SHARC (Surface Hopping including ARbitrary Couplings) method, ${ }^{16-18}$ where the nuclei are treated classically and the electrons quantum mechanically. Despite the mixed quantum-classical nature of surface-hopping methods ${ }^{19}$, SHARC has proved to be particularly successful to investigate the role of intersystem crossing dynamics in nucleobases, ${ }^{20-24}$ nucleobase analoga, ${ }^{25-27}$ and other molecular systems. ${ }^{28-32}$ SHARC is also suited to simulate time-resolved photo-electron spectroscopy. ${ }^{15}$

\subsection{Electronic state representations}

In the discussion of electronic states, it is important to clearly define the different bases in which these states can be represented. For example, the terms "diabatic" and "adiabatic" electronic states might be misleading in the presence of SOCs, and care should be taken 
in the adopted nomenclature. "Adiabatic" literally means "impassable", and with respect to electronic states, it can be associated with potential energy surfaces (PESs) which cannot pass (cross) through each other. Since this is a rather imprecise definition, mathematically, adiabatic states are obtained as the eigenstates of the electronic Hamiltonian

$$
\hat{H}^{\mathrm{el}}\left|\Psi_{\beta}^{\text {adiab }}\right\rangle=E_{\beta}^{\mathrm{el}}\left|\Psi_{\beta}^{\text {adiab }}\right\rangle,
$$

where $E_{\beta}^{\mathrm{el}}$ is the electronic energy of state $\beta$. In the simplest case, a non-relativistic electronic Hamiltonian is considered with only one spin multiplicity and where no external field is applied. In this case, the Hamiltonian contains only kinetic energy and Coulombic terms of the molecular system:

$$
\hat{H}^{\mathrm{MCH}}=-\sum_{i}^{n_{\mathrm{el}}} \nabla_{i}^{2}+\sum_{A<B}^{n_{\mathrm{nuc}}} \frac{Z_{A} Z_{B}}{\left|\vec{R}_{A}-\vec{R}_{B}\right|}-\sum_{i}^{n_{\mathrm{el}}} \sum_{A}^{n_{\mathrm{nuc}}} \frac{Z_{A}}{\left|\vec{R}_{A}-\vec{r}_{i}\right|}+\sum_{i<j}^{n_{\mathrm{el}}} \frac{1}{\left|\vec{r}_{i}-\vec{r}_{j}\right|},
$$

where $i, j$ and $A, B$ are the electron and nuclear indices, respectively, $Z$ are the nuclear charges, and $\vec{r}$ and $\vec{R}$ are the electron and nuclear positions, respectively. Following the nomenclature of Mai et al., ${ }^{17,21}$ we term this Hamiltonian the Molecular Coulomb Hamiltonian ( $\left.\mathrm{MCH}\right)$, because there are no external fields (only the molecule) and no interactions beyond the Coulomb ones. Most quantum chemistry calculations yield electronic states which are eigenstates of this $\mathrm{MCH}$, which could be called "MCH states" (see Fig. 1b), or "states in the MCH picture". Within each multiplicity, these states do not cross each other (i.e., they are adiabatic), and from their energetic ordering and spin multiplicity it is sensible to denote the states as $S_{0}, S_{1}$, $\ldots, T_{1}, T_{2}$, etc.

\section{[Figure 1 about here.]}

When more than one multiplicity is considered simultaneously and states couple through the relativistic SOCs, the situation is more complicated. In this case, the electronic Hamiltonian is the sum of the $\mathrm{MCH}$ and the SOC Hamiltonian

$$
\hat{H}^{\mathrm{el}}=\hat{H}^{\mathrm{MCH}}+\hat{H}^{\mathrm{SOC}},
$$

where the $\mathrm{MCH}$ might or might not contain relativistic corrections to the electron kinetic energy. SOCs introduce off-diagonal terms in the electronic Hamiltonian which couple states of different spin multiplicity. More details of SOCs-their relativistic origin, their mathematical form, and methods for obtaining ab initio values-are discussed in section 10.4.3. Here, we only want to stress that SOCs lift the block-diagonal form of the $\mathrm{MCH}$, and hence it is not sufficient anymore to consider only a single multiplicity. In this context, adiabatic states could be obtained as the eigenstates of this full electronic Hamiltonian. However, computing such eigenstates-i.e., relativistic quantum chemistry-can be extremely demanding. ${ }^{33,34}$ Consequently, in most quantum chemistry calculations only $\mathrm{MCH}$ states of different multiplicities (e.g., singlets and triplets) are computed, with SOCs considered as a perturbation. Even though these $\mathrm{MCH}$ states of different multiplicities are used as electronic basis, they are not eigenstates of the full Hamiltonian. Approximate eigenstates of the full Hamiltonian can instead be obtained by constructing the matrix representation of the electronic Hamiltonian 
in the space of a small number of relevant $\mathrm{MCH}$ states, denoted as $\mathbf{H}^{\mathrm{MCH}}$ (note that the superscript refers to the representation of the matrix) and containing elements

$$
H_{\beta \alpha}^{\mathrm{MCH}}=\left\langle\Psi_{\beta}^{\mathrm{MCH}}\left|\hat{H}^{\mathrm{el}}\right| \Psi_{\alpha}^{\mathrm{MCH}}\right\rangle,
$$

and diagonalizing it. To avoid misunderstanding, and because these states originate from a diagonalization, we term the basis of the eigenstates of the electronic Hamiltonian including all perturbative terms the "diagonal" basis (see Fig. 1c). The resulting states do not cross each other (i.e., they are adiabatic), but each state is a mixture of different multiplicities and therefore they can only be denoted as diagonal state $1,2,3$, etc.

The electronic states can also be represented in a so-called diabatic picture, where the states are defined such that they have a constant electronic character for all coordinates (e.g., ${ }^{1} \pi \pi^{*}$, see Fig. 1a). The advantage of such a picture is that most spectroscopic properties, like the corresponding transition dipole moments, stay relatively constant for different molecular conformations. Therefore, experimental observables often directly relate to diabatic states, e.g., a bright $\pi \pi^{*}$ state can be detected while the dark $n \pi^{*}$ state does not give a (strong) signal. Because spectroscopic observations can be readily interpreted and discussed in terms of such diabatic states, we also label the diabatic representation "spectroscopic representation". ${ }^{17,21}$ Yet, one should keep in mind that a rigorous diabatic representation cannot be defined for polyatomic molecules. ${ }^{35,36}$ Even approximate diabatic states are not suitable for computations because a very large number of diabatic states is necessary to describe all parts of the PESs accurately. Hence, quantum chemical computations are generally carried out in the either the $\mathrm{MCH}$ or the diagonal representation. Within SHARC, we employ quantum chemical computations in the $\mathrm{MCH}$ representation, which are converted to the diagonal one. Hence, it is not necessary to obtain diabatic states to carry out SHARC simulations; instead, diabatic states are only employed for a posteriori analysis purposes, if they are available.

It is very instructive to see the influence of the choice of representation on the terms appearing in the nuclear Schrödinger equation:

$$
\sum_{\alpha}[\hat{T}^{\mathrm{n}}-\sum_{A}^{n_{\text {nuc }}} \frac{1}{2 M_{A}} \underbrace{\left\langle\Psi_{\beta}\left|\vec{\nabla}_{A}\right| \Psi_{\alpha}\right\rangle}_{\vec{T}_{\beta \alpha}} \vec{\nabla}_{A}+\underbrace{\left\langle\Psi_{\beta}\left|\hat{H}^{\mathrm{el}}\right| \Psi_{\alpha}\right\rangle}_{H_{\beta \alpha}}]\left|\chi_{\alpha}\right\rangle=\mathrm{i} \hbar \frac{\partial}{\partial t}\left|\chi_{\beta}\right\rangle,
$$

where $\hat{T}^{\mathrm{n}}$ is the nuclear kinetic energy operator, $M_{A}$ is the mass of atom $A$, and the $\left|\chi_{i}\right\rangle$ is the nuclear wave function for state $i$ (and where the diagonal Born-Oppenheimer terms are omitted). In this equation, $\vec{T}_{\beta \alpha}$ are the nonadiabatic coupling (NAC) vectors and $H_{\beta \alpha}$ are the matrix elements of the electronic Hamiltonian. These two terms directly depend on the choice of representation, i.e., the choice of the electronic states $\left|\Psi_{\alpha}\right\rangle$, and the electronic Hamiltonian. Considering a Hamiltonian including SOCs, in the MCH representation neither $\vec{T}_{\beta \alpha}$ nor $H_{\beta \alpha}$ have any special form. In contrast, in the diagonal representation, the matrix of the $H_{\beta \alpha}$ is diagonal, while in the diabatic representation, the $\vec{T}_{\beta \alpha}$ terms vanish.

From the discussion above one can infer that while computational results are usually discussed in either the $\mathrm{MCH}$ or diagonal representation, experiments are typically analyzed in terms of spectroscopic states. Therefore, care should be taken with these two different 
nomenclatures, because in general it is not possible to directly correlate $\mathrm{MCH}$ and spectroscopic (or diabatic) states. Even the simple label of " $S_{1}$ " can be confusing when the order of the states changes at different geometries. The " $S_{1}$ " label is sometimes used to refer to the state that most closely resembles the electronic character of the $S_{1}$ at the Franck-Condon geometry or, alternatively, to the second-lowest $\mathrm{MCH}$ state at the geometry of interest, no matter the character. We argue that the latter is more appropriate and one should prefer more descriptive state labels, such as ${ }^{1} \pi \pi^{*},{ }^{1} n \pi^{*},{ }^{1} L_{a}, \ldots$, when one is actually talking about a state of a particular electronic character.

These different representations are relevant in an analogous way if the states interact via dipole moment-field couplings (DFCs), when the interaction with the laser field is explicitly taken into account (see Fig. 1d-f). Note that in this case, the diagonal states are time-dependent due to the time-varying electric field.

Which representation to use is not only a matter of taste or convenience, but it is relevant in computational simulations as the different representations are only equivalent when using a fully quantum-mechanical description for both nuclei and electrons. However, this equivalence is lost when classical approximations are employed. In the following, we shall introduce the SHARC method, which is a mixed quantum-classical method in which the choice of representation is determinant.

\subsection{Nonadiabatic dynamics: SHARC}

Before discussing the strengths and weaknesses of SHARC, it is instructive to briefly explain a few general concepts of nuclear dynamics simulations, starting from the nuclear Schrödinger equation, from quantum to mixed quantum-classical dynamics, with particular emphasis on surface-hopping. Then, the special aspects of SHARC are introduced.

A method useful to perform photodynamics simulations in the ultrafast domain (fs to ps) has to comply with several important requirements. First, in order to describe ultrafast processes, which are always very far from any equilibrium, it is necessary to explicitly treat the motion of all constituent particles-nuclei and electrons. Approaches such as Fermi's golden rule ${ }^{37}$ or nonadiabatic transition state theory, ${ }^{38}$ used in the study of (slow) excited-state processes, are not applicable to the ultrafast time regime.

Second, the method should consider the actual PESs involved, because approximate analytical potentials, e.g., harmonic ones, might fail to describe relevant parts of the conformational space.

Third, the method should describe the interaction between several different electronic states, i.e., diabatic or nonadiabatic interactions, explicitly. This is essential for the description of excited-state dynamics as excited states are often not well separated in energy, ${ }^{39}$ and therefore show an increased propensity for population transfer. ${ }^{40}$ Related to this point is the requirement that the method should be able to account for any kind of coupling between the electronic states, in order to allow the consistent simulation of different types of ultrafast processes, e.g., internal conversion (IC) or intersystem crossing (ISC).

And finally, in order to be applicable to physically and chemically relevant problems, the method needs to be able to describe the nuclear motion of large (i.e., more than a dozen atoms) molecules in a practical way. 


\subsubsection{From quantum dynamics to molecular dynamics}

In principle, the nuclear motion of an excited molecule can be exactly described by solving the nuclear time-dependent Schrödinger equation, as given in Eq. (5). This equation can be derived ${ }^{41,42}$ from the total time-dependent Schrödinger equation ${ }^{43,44}$ of the system by inserting the Born-Huang expansion for the total molecular wave function and assuming that one has found suitable solutions of the electronic Schrödinger equation (Equation (1)).

Solving the nuclear time-dependent Schrödinger equation numerically is usually called quantum dynamics or wave packet dynamics. ${ }^{45}$ It is the most accurate method for the simulation of nuclear dynamics and in combination with accurate electronic energies it is able to match the accuracy of many experiments. ${ }^{45}$ In order to solve the nuclear time-dependent Schrödinger equation, it is necessary to first compute the PESs of all relevant electronic states over all relevant parts of the conformational space. Unfortunately, the conformational space grows exponentially as the number of degrees of freedom goes up. This means that already for five-atomic molecules, standard quantum dynamics becomes essentially unfeasible. ${ }^{45}$ Using the related multi-configurational time-dependent Hartree (MCTDH) method, it is possible to treat more dimensions, ${ }^{46}$ but even in this case the bottleneck of computing high-dimensional PESs remains. Hence, these quantum dynamics methods can only be applied to rather small systems in the gas phase, or to large systems if some degrees of freedom are neglected. Consequently, different approaches with different approximations (possibly neglecting some quantum effects) in the computations have been developed, see e.g. Refs. 17,45,47-57.

One of the most ubiquitous approximations to deal with many degrees of freedom is to treat the nuclear motion classically. In this way, each atom can be described as a massive point charge moving according to Newton's equation of motion, instead of having to deal with the large, multi-dimensional nuclear wave function. Furthermore, Newton's equation requires only local information on the PES-namely, the gradient of the potential energy-and thus can be easily combined with on-the-fly ${ }^{58}$ computations. In this way, the a priori calculation of the PES can be fully avoided. An additional advantage of classical nuclear dynamics is that because it can be easily propagated in Cartesian coordinates, it is not necessary to define internal coordinates or select the few most important modes where to propagate the nuclear motion. Compared to QD, classical molecular dynamics (MD) is much less restricted in the number of atoms or the time scales, as shown by the large number of bio-molecular applications to systems of hundreds of thousands of atoms and time scales of many nanoseconds in the electronic ground state. However, for excited-state dynamics, the limitations in system size and time scale remain as they are primarily linked to solving the electronic problem.

\subsubsection{From Born-Oppenheimer molecular dynamics to surface hopping}

Unfortunately, classical MD fails to describe a number of quantum effects. ${ }^{19,57}$ (i) As classical MD (i.e., Born-Oppenheimer MD) considers only one potential energy, it neglects all nonadiabatic interactions between different electronic states. Consequently, it is not possible to describe photophysical processes like IC and ISC. (ii) As all nuclei are classical, point-like particles, it is not possible to simulate the splitting of a wave packet into multiple reaction pathways. This is usually not a problem for long ground state simulations close to equi- 
librium, but for non-equilibrium processes like in photodynamics, the simulated trajectory will randomly follow only one of the available reaction pathways. This problem is usually mitigated by considering an ensemble of independent trajectories and statistically analyzing the branching of the trajectories into the different pathways. (iii) However, even though a trajectory ensemble recovers the splitting behavior of the wave packet, classical MD cannot describe (de-)coherence and interference of different parts of the wave packet. ${ }^{57}$ This is primarily because the trajectories in the ensemble are independent of each other. (iv) Classical mechanics cannot properly represent the effects of the zero-point energy (ZPE). The ZPE is a consequence of the Heisenberg uncertainty principle and requires that even in the vibrational ground state, each normal mode retains a certain amount of energy. However, in classical MD it is possible that the nuclear motion completely stops and no vibrational energy is retained. Furthermore, the vibrational energy can freely flow between the degrees of freedom. (v) Finally, classical MD is not able to account for tunneling through classically forbidden energy barriers.

Certainly, for the description of excited-state dynamics, the presence of nonadiabatic interactions between the electronic states is essential (recall point (i)). In order to extend classical MD to excited states, there are two main approaches in use: Ehrenfest dynamics ${ }^{51}$ and surface hopping (SH). ${ }^{47,59}$ Both approaches are based on simulating a total electronic wave function, which is propagated along with the classical nuclei. The total electronic wave function $\Psi^{\mathrm{el}}(t)$ is described as a linear combination of the different electronic states $\Psi_{\alpha}^{\mathrm{el}}(t)$ :

$$
\Psi^{\mathrm{el}}(t)=\sum_{\alpha} c_{\alpha}(t) \Psi_{\alpha}^{\mathrm{el}}(t)
$$

By inserting this equation into the time-dependent Schrödinger equation, one can derive the equation of motion (EOM) for the coefficients $c_{\alpha}(t):{ }^{42}$

$$
\frac{\partial}{\partial t} c_{\beta}(t)=-\sum_{\alpha}\left[\mathrm{i} H_{\beta \alpha}+\vec{v} \cdot \vec{T}_{\beta \alpha}\right] c_{\alpha}(t),
$$

where $H_{\beta \alpha}$ is an element of the electronic Hamiltonian matrix, which contains the electronic energies on the diagonal and coupling terms on the off-diagonal. Furthermore, $\vec{v}$ is the nuclear velocity vector and $\vec{T}_{\beta \alpha}$ is a NAC vector $\left(\vec{T}_{\beta \alpha}=\left\langle\Psi_{\beta}|\vec{\nabla}| \Psi_{\alpha}\right\rangle\right)$. Both kinds of coupling terms will be discussed in more detail below. This EOM allows propagating the coefficients from a time step $t$ to the next time step, given the energies and coupling terms of all electronic states.

Naturally, the evolution of the electronic wave function should have an effect on the nuclear dynamics, hence some coupling scheme has to be introduced. The two approaches, Ehrenfest dynamics and SH, differ in how this coupling is accomplished, i.e., in the way the potential energy and gradients are derived from the electronic wave function. In Ehrenfest dynamics, the potential energy is the average of the energies of the states, weighted by the amplitudes $\left|c_{\alpha}(t)\right|^{2}$. In this way, when the electronic wave function changes due to nonadiabatic interactions, the energy and gradients adapt to the new electronic situation. However, if several amplitudes are large, Ehrenfest dynamics can lead to unphysical average trajectories, which do not follow either of two reaction pathways, but the average of them. ${ }^{42}$

The SH approach ${ }^{47,59}$ has become very popular in the last decades, which explains the proliferation of excellent reviews on the topic. ${ }^{19,42,57,60} \mathrm{In} \mathrm{SH}$, the potential energy at each 
time step is always one of the energies of the considered electronic states. At each time step, based on the evolution of the amplitudes $\left|c_{\alpha}(t)\right|^{2}$ the method determines stochasticallyby drawing a random number-which of the electronic states is the "active" state. If the algorithm decides to change the active state, a "surface hop" is performed, which is the origin of the method's name. In order to conserve total energy, the nuclear velocities are rescaled during such a surface hop. Since the potential energy is always the energy of one of the electronic states, $\mathrm{SH}$ fully avoids the nonphysical average trajectories encountered in Ehrenfest dynamics. However, the stochastic nature of the algorithm requires a sufficiently large ensemble of independent trajectories, in order to produce the correct branching ratios during a nonadiabatic event. Hence, the $\mathrm{SH}$ method solves problems (i) and (ii) explained above.

Concerning point (iii), many SH simulations employ some kind of empirical or ad hoc decoherence correction schemes. ${ }^{57,61-63}$ These are necessary, since a given trajectory might have large amplitudes $\left|c_{\alpha}(t)\right|^{2}$ for several states, but the nuclear motion follows only one of them. In principle, this is unphysical because these other amplitudes are artificially dragged along with the active state, i.e., the states are too coherent. In reality, the amplitudes of different states would follow their individual gradients and quickly separate. Therefore, in most decoherence correction schemes the amplitudes of the non-active states are damped and the amplitude of the active state rescaled such that the total population is conserved. While this might seem like a rather technical detail, the decoherence correction has a quite palpable effect: for an ensemble of trajectories, it makes the sums of the amplitudes (the "quantum" populations of the ensemble) consistent with the fraction of trajectories in each state (the "classical" populations of the ensemble).

Figure 2 illustrates the differences between a quantum dynamics simulation and an $\mathrm{SH}$ simulation. In the former (panel (a)), a Gaussian wave packet (initially in light gray on the upper left) moves through an avoided crossing, is mostly transferred to the lower adiabatic state, and continues to travel to the right. Is is apparent that some parts of the wave packet are also reflected around the avoided crossing and can be found on the lower left part of the potential curve. In the SH simulation (panel (b)), each line represents one trajectory, all starting on the upper left. Most trajectories perform one surface hop to the lower state and continue to the right, just as in the wave packet simulation. A couple of trajectories instead get reflected before hopping, and end up on the lower left part.

\section{[Figure 2 about here.]}

Unfortunately, regular SH still misses other quantum effects. As all trajectories are completely independent, it is not possible to simulate interference when different parts of the ensemble would meet each other in phase space. The problem of interference (iii) can be solved by methods which go beyond regular $\mathrm{SH}$, like multiple spawning ${ }^{64}$ or multiple cloning ${ }^{65}$; these methods are computationally more demanding than SH as they use ensembles of coupled trajectories. However, $\mathrm{SH}$ remains a valid method choice when interferences do not play a significant role, for example in large molecules, where it might become increasingly unlikely that separated parts of the wave packet ever meet again. ${ }^{66,67}$

Regarding the treatment of ZPE (iv), there exists no rigorous and at the same time practical (for large systems) solution to this problem. ${ }^{68}$ A pragmatical solution for $\mathrm{SH}$ simulations is 
to generate initial conditions with as much initial energy as the $\mathrm{ZPE}^{69}$ and check that no significant errors accumulate during the propagation.

The problem of including tunneling (v) is also very difficult to treat because in $\mathrm{SH}$ the potential energies are usually computed on-the-fly, i.e., only local information about the PES is known. Thus, it is not possible to identify in advance energy barriers which could be tunneled through. Such identification would add many energy calculations per time step to scan forward and obtain energy profiles to compute tunneling probabilities. Alternatively, it is possible to allow tunneling only for particular degrees of freedom..$^{70}$ Another solution to this problem is to use ring-polymer molecular dynamics (RPMD), ${ }^{54,71}$ where each nucleus is treated as a set of several coupled point-like masses, which can exchange energy and therefore might be able to climb classically forbidden barriers. As there is no free lunch, RPMD requires many more energy calculations than regular $\mathrm{MD}$ or $\mathrm{SH}$, so it is only employed if tunneling is known to be a critical part of the investigated process.

Notwithstanding the fact that these quantum effects cannot be described by SH, this method is still extremely useful for many applications. In particular, $\mathrm{SH}$ is computationally very efficient to treat the excited-state dynamics of molecular systems with many degrees of freedom, due to its on-the-fly and independent-trajectories paradigms, which reduce the required number of energy calculations and allow for a trivial parallelization of the computational workload for multiple trajectories. ${ }^{19}$

\subsubsection{From Surface Hopping to SHARC}

The original formulation of $\mathrm{SH}^{47,59}$ is targeted only at the description of IC. In the basis of the eigenstates of the $\mathrm{MCH}$, IC is mediated by the NAC vectors between the excited states. These NAC vectors enter the EOM for the electronic wave function coefficients (see equation (7)) and lead to transfer of population from one state to another.

In order to describe other kinds of nonadiabatic processes-absorption, stimulated emission, or ISC-additional couplings have to be introduced in the EOM. For absorption and stimulated emission, the explicit interaction of the system with an electric field is necessary. The most basic interaction term is the scalar product of the molecular (transition) dipole moment matrix with the electric field vector $\overrightarrow{\mathcal{E}}$. These DFCs are then included in the electronic Hamiltonian matrix elements in the EOM (equation (7)) as

$$
H_{\beta \alpha}=\delta_{\beta \alpha} E_{\beta}+\left\langle\Psi_{\beta}\left|-\sum_{i}^{n_{\mathrm{el}}} \vec{r}_{i}+\sum_{A}^{n_{\text {nuc }}} Z_{A} \vec{R}_{A}\right| \Psi_{\alpha}\right\rangle \cdot \overrightarrow{\mathcal{E}},
$$

and allow an electric field to modify the electronic populations.

The description of ISC requires SOCs (see Section 10.4.3 for more details) to be included in the electronic Hamiltonian elements of the EOM (equation (7)) as

$$
H_{\beta \alpha}=\delta_{\beta \alpha} E_{\beta}+\left\langle\Psi_{\beta}\left|\hat{H}^{\mathrm{SOC}}\right| \Psi_{\alpha}\right\rangle .
$$

SOC is a relativistic effect and hence, ${ }^{37}$ the most accurate way to proceed would be to compute fully relativistic four- (or two-)component electronic states ${ }^{33,34}$ and perform regular $\mathrm{SH}$ on the resulting potentials. In such relativistic calculations, states of different multiplicity (e.g., 
singlets and triplets) are automatically mixed, and the coupling between these mixed states is described by terms analogous to the regular NAC vectors. Hence, no modifications to the $\mathrm{SH}$ algorithm, besides using a relativistic electronic structure method, would be required. In such a simulation, IC and ISC would be indistinguishable, as they are both mediated by NACs between the relativistic electronic states. However, such relativistic calculations are nowadays computationally very demanding and the implementations of relativistic excited states gradients and NAC vectors are not yet developed enough ${ }^{72}$ to be applied to nonadiabatic dynamics simulations.

An alternative to fully relativistic calculations is the calculation of spin-orbit mixed states through a perturbative scheme. ${ }^{73,74}$ In this ansatz, one first computes a set of $\mathrm{MCH}$ states (e.g., a small set of singlet and triplet states relevant for the investigated process, see section 10.2). For this set of states, the full electronic Hamiltonian matrix in the basis of the MCH states, $\mathbf{H}^{\mathrm{MCH}}$ (equation (4)) is computed and diagonalized to yield approximate relativistic states:

$$
\mathbf{H}^{\text {diag }}=\mathbf{U}^{\dagger} \mathbf{H}^{\mathrm{MCH}} \mathbf{U} \text {. }
$$

Here, the matrix $\mathbf{H}^{\text {diag }}$ is a diagonal matrix containing the approximate energies of the spinorbit mixed states, called diagonal states (see section 10.2). The approximation in this scheme lies in neglecting all spin-orbit couplings between the included small set of states and all other states. For systems without very heavy atoms (i.e., atoms with filled $6 p$ orbitals and higher ${ }^{75}$ ), this ansatz usually produces accurate spin-orbit states, while being significantly cheaper than two- or four-component relativistic methods.

The main idea behind SHARC ${ }^{16,17}$ is to perform SH on the potentials obtained through diagonalization, as shown in equation (10). For problems including ISC, this ansatz will hence use the approximate spin-orbit-mixed states discussed above. Equivalently, the DFC matrix can be included in the diagonalization to obtain field-dressed states on which the propagation can be performed. ${ }^{76}$

The diagonalization of the electronic Hamiltonian has certain advantages ${ }^{17,77,78}$ over using the (SOC or DFC) interaction matrix directly in the EOM for the electronic wave function. First, in the case of ISC dynamics, the diagonalization leads to rotation-invariant states, which means that the sum of the population transfer to the component states of a multiplet is independent of the orientation of the molecule or the definition of the spin functions. ${ }^{17,78}$ Not using such a diagonalization scheme instead would lead to ISC rates which depend on the orientation or the spin functions. Second, SOCs (in ISC dynamics) or DFCs are usually of almost constant magnitude over a large portion of the PESs, and therefore can induce hops in a large phase space volume. ${ }^{47,78,79}$ This is disadvantageous for SH simulations, since a large number of trajectories is required to sample in an unbiased way these hops. Upon diagonalization, the delocalized SOCs/DFCs are replaced by localized NAC-like terms which are large only where the energy gap between the corresponding states is small. By localizing the coupling terms, a significantly reduction in the amount of trajectories is achieved.

The diagonalization step in SHARC requires several modifications of the original SH algorithm. First, it is necessary to numerically control the form of the transformation matrix $U$, which can contain arbitrary signs or phase factors which lead to non-smooth propagation. ${ }^{17,80}$ Second, the propagation in the diagonal basis modifies the EOM of the electronic coefficients, 
which includes the transformation matrix $\mathbf{U}$ :

$$
\frac{\partial}{\partial t} \vec{c}(t)=\left[\mathrm{iU}^{\dagger} \mathbf{H}^{\mathrm{MCH}} \mathbf{U}+\mathbf{U}^{\dagger}\left(\vec{v} \overrightarrow{\mathbf{T}}^{\mathrm{MCH}}\right) \mathbf{U}+\mathbf{U}^{\dagger} \frac{\partial}{\partial t} \mathbf{U}\right] \vec{c}_{\alpha}(t)
$$

This equation could be solved by directly integrating:

$$
\vec{c}^{\operatorname{diag}}(t+\Delta t)=\exp \left[-\int_{t}^{t+\Delta t}\left(\mathrm{i}^{\dagger} \mathbf{H}^{\mathrm{MCH}} \mathbf{U}+\mathbf{U}^{\dagger}\left(\vec{v} \overrightarrow{\mathbf{T}}^{\mathrm{MCH}}\right) \mathbf{U}+\mathbf{U}^{\dagger} \frac{\partial}{\partial t} \mathbf{U}\right) \mathrm{d} \tau\right] \vec{c}^{\operatorname{diag}}(t),
$$

but this would involve differentiation and integration of $\mathbf{U}$, a matrix which can change locally extremely fast. ${ }^{17}$ Instead, in SHARC the coefficients are obtained through:

$$
\vec{c}^{\operatorname{diag}}(t+\Delta t)=\mathbf{U}^{\dagger}(t+\Delta t) \exp \left[-\int_{t}^{t+\Delta t}\left(\mathrm{iH}^{\mathrm{MCH}}+\left(\vec{v} \overrightarrow{\mathbf{T}}^{\mathrm{MCH}}\right)\right) \mathrm{d} \tau\right] \mathbf{U}(t) \vec{c}^{\operatorname{diag}}(t) .
$$

Here, differentiation and integration of $U$ are simply replaced by matrix products involving the $\mathrm{U}$ of the previous and current time step, a practice which was inspired by the localdiabatization method. ${ }^{81}$ This scheme is numerically very stable, especially if SOCs are small. Third, as the SHARC dynamics is carried out on the energies in the diagonalized Hamiltonian, it is necessary to find the gradients of these diagonal energies. ${ }^{17}$ These gradients can be computed as a linear combination of the gradients and NAC vectors of the MCH states, meaning that at each time step it is necessary to compute multiple $\mathrm{MCH}$ gradients, which increases the computational effort compared to regular SH. Despite appearing technical, these modifications are essential to correctly perform $\mathrm{SH}$ in the diagonal representation, as recognized by recent further implementations of dynamics codes using a diagonalized Hamiltonian. ${ }^{80}$

\subsubsection{Practical aspects of SHARC simulations}

One important aspect of carrying out a SHARC (or any other SH) simulation is the preparation of the initial conditions. To find the relaxation pathway after vertical excitation from the ground state, the initial conditions are usually chosen to resemble the nuclear phase space distribution in the equilibrium of the ground state. In such a case, there are two main approaches to sample the ground state positions and momenta. ${ }^{69,82}$ In the first, one computes the harmonic frequencies and normal modes of the molecule at the equilibrium geometry and picks position/momentum snapshots from the Wigner distribution of the harmonic oscillator model, ${ }^{83,84}$ possibly including temperature effects by considering the Wigner distribution of higher vibrational states. This approach is very useful for relatively small and stiff molecules in vacuum, as it generates a good representation of the ZPE of the system. For larger systems, this approach might not be appropriate if the harmonic oscillator model becomes a bad approximation due to the presence of strongly anharmonic or non-linear modes, or if the approach becomes impractical due to the high computational cost of calculating the Hessian. In such cases, one resorts to a classical MD simulation in the ground state and picks random position/momentum snapshots from the resulting trajectory. ${ }^{85}$ The disadvantage of the latter method is that each degree of freedom receives the same share of the thermal energy, even 
though in a quantum mechanical distribution high-frequency modes should receive more energy than low-frequency ones. ${ }^{69}$

After finding appropriate initial positions and momenta, it is also necessary to determine the correct initial electronic state. This step is related intimately to the way the molecule is experimentally pumped to the electronic excited state. In many experiments, a good temporal resolution requires that the pump pulse is as short as possible. Consequently, in the preparation of initial conditions for SHARC, one routinely assumes an infinitely short laser pulse (a delta pulse) and simply excites the snapshots vertically. Often, the initial electronic state is chosen stochastically based on the oscillator strength of all states; ${ }^{86}$ in this way, only bright states are initially populated, as it would be the case in a typical experiment. An alternative to the delta pulse approach is to initiate the dynamics simulation in the ground state and explicitly include the pump pulse in the SHARC simulation. This approach is significantly more expensive, as the laser pulse might excite only a small fraction of the ground state trajectories and the remaining ones will be wasted.

Also important for SHARC, and SH in general, is the number of trajectories. ${ }^{19}$ Ideally, a number of criteria should be satisfied. First, for non-equilibrium classical MD simulations, one should employ multiple trajectories to sample different reaction pathways. The number of trajectories should therefore be such that the initial wave function is adequately represented, covering the relevant phase space volume. Second, in excited state dynamics, the ensemble should additionally consider that the initial conditions are distributed over multiple electronic states, depending on the laser bandwidth. And third, specific to $\mathrm{SH}$ simulations, one also needs multiple trajectories to sample the stochastic hopping events for each nonadiabatic event. Hence, optimally one generates a phase-space distribution of initial positions/momenta, and for each position one would generate a distribution over the relevant electronic states, and for each initial state one would generate multiple trajectories with different random number sequences. The actual number of trajectories then also depends on whether one is interested only in the main relaxation pathways or if the main focus is on one of the side channels; in the latter case more trajectories are required for adequate statistics.

In practice, however, these suggestions for the number of trajectories can rarely be met to full extent. One is usually limited by the computational expense of the trajectories, which primarily depends on the choice of the electronic structure method used to compute energies, gradients, and coupling terms on-the-fly. Only for very cheap methods or when using precomputed PESs, it is possible to simulate thousands of trajectories; when using ab initio electronic structure methods not more than a few hundred trajectories are typically computed in practice. Such a small number of trajectories might increase the statistical noise in the extracted properties of interest, but this is often preferable to employing a cheaper electronic structure methods which yields unphysical potentials. Therefore, it is always necessary to find an acceptable compromise between computational effort and accuracy of the electronic structure calculations. As these calculations are a topic of high importance for any SHARC (or $\mathrm{SH}$ ) simulation, it is discussed in detail in the next section. 


\subsection{Electronic structure methods}

The electronic structure computations underlying SHARC (or SH) simulations have to provide three general types of ingredients: (i) energies and gradients of the individual electronic states, (ii) coupling terms between the states, and (iii) terms needed to simulate experimental observables from various probing techniques. Point (i) is formally satisfied by any method that can perform excited-state structure optimizations, which is a large subset of the methods which can compute excitation energies. For (ii), three types of coupling terms are usually considered within SHARC (see above): NACs leading to IC between states of the same spin, SOCs leading to ISC between states of different spin, and DFCs governing the interaction of a molecule with an external electric field. Regarding (iii), SHARC can also incorporate further electronic quantities to simulate experimental observables. Currently, SHARC is able to incorporate transition dipole moments-allowing the simulation of transient absorption spectra-and Dyson norms, to approximate ionization probabilities.

The choice of the electronic structure level of theory-which provides all electronic quantities during the on-the-fly trajectory simulation-is usually the parameter that most strongly affects the outcome of the simulations. A poor choice, such that critical aspects of the PESs are not described correctly, can lead to meaningless dynamics.

\subsubsection{Excited-state energies and gradients}

The right choice of electronic structure method to calculate excited-state energies is particularly challenging when electronic states with different wave function characters participate in the dynamics. Valence states $\left(n \pi^{*}, \pi \pi^{*}\right.$, ones involving $\sigma$ orbitals), Rydberg states, charge transfer states, etc., often demand different electronic structure descriptions. Hence, it might be not trivial to find a method which describes then all on the same footing.

A good practice is to validate the electronic structure method before starting the dynamical simulations. A starting point is to compare the excitation energies and state ordering at the Franck-Condon geometry with a reliable level of theory or to experimental results. However, as the trajectories will quickly leave the Franck-Condon region and travel to other parts of the PESs, this validation approach is actually of limited value. It is strongly advisable that geometries likely critical for the dynamics-excited-state minima, minimum-energy crossing points, dissociation limits, etc.-are optimized prior to the dynamics simulations. Only if the chosen electronic structure method can appropriately describe the energies at these geometries, the method can be expected to be reliable for dynamics.

While the accuracy of the electronic structure method is of fundamental importance, the choice of method is also restricted by the computational cost, as SH simulations require up to hundreds of thousands of single point calculations. The need to simulate long time scales and the available resources can also limit feasible options. Therefore, the choice of the electronic structure method has to be balanced between the available resources and the desired accuracy. In the following, we describe briefly methods which are commonly used in SH simulations. The methods for which interfaces are available in SHARC are collected in Table 1.

[Table 1 about here.] 
For general applicability, a method also needs to correctly and efficiently treat challenging electronic structure situations that lead to unpaired electrons in the ground state, such as ground state/excited state crossings, bond breaking, or strong molecular deformations. In this situation, the ground state electronic structure can no longer be approximated by a single electronic configuration and one should resort to multi-configurational methods. ${ }^{87}$ The complete active space self-consistent field (CASSCF) method, ${ }^{88}$ which allows optimizing the orbitals for multi-configurational wave functions, offers a logical starting point. It has indeed been used for a wide range of dynamics studies including nucleobases and analogues, ${ }^{20,22,24,89-91}$ unsaturated hydrocarbons, ${ }^{92-94}$ or various aromatic systems. ${ }^{29,32,95-99}$ The downside of CASSCF is that it does not include dynamic electron correlation and thus tends to provide an imbalanced description of excited states of different character, e.g., $n \pi^{*}$ and $\pi \pi^{*}$.

A correlated multi-reference description is provided by multi-reference configuration interaction (MRCI) ${ }^{100}$ or CAS perturbation theory (CASPT2). ${ }^{101}$ In the context of SHARC simulations, the MRCI method has been applied to a number of small and medium-sized systems, including nucleobases, ${ }^{27,102-105}$ unsaturated hydrocarbons, ${ }^{106,107}$, pyrrole, ${ }^{108}$ and $\mathrm{SO}_{2} \cdot{ }^{28}$ CASPT2 in its uncontracted or contracted forms is seldom employed in dynamics simulations ${ }^{26,109}$ due to its high cost. A promising new implementation of extended multistate CASPT2 for nonadiabatic dynamics simulations has been reported recently. ${ }^{110}$ Though MRCI and CASPT2 are among the most accurate methods, they also have downsides: MRCI lacks size-extensivity ${ }^{87}$-leading to a degradation of its accuracy as system size increases-and CASPT2 is rather sensitive to empirical shift parameters. ${ }^{111}$ Furthermore, all multi-reference methods suffer from the fact that the results crucially depend on the choice of the active orbital space.

For the above reasons, there is a strong drive to use single-reference methods that can offer much better computational performance while retaining sufficient accuracy. ${ }^{112}$ However, care must be taken at strongly distorted geometries where the ground state and excited states become near-degenerate, because single-reference methods might completely fail in this situation, either by exhibiting serious convergence problems (which might lead to the abort of the trajectory) or by describing an unphysical topology of the ground state-excited state conical intersection. In contrast, conical intersections between two excited states are usually ${ }^{113}$ described with the correct topology. Hence, single-reference methods are preferably used for nonadiabatic dynamics where the ground state is not involved.

Among single-reference methods, time-dependent density functional theory (TD-DFT) is attractive due to its computational efficiency. Applications include transition metal complexes, ${ }^{31,114,115}$ nucleobases (see, e.g., citations in Ref. 91), and larger biological molecules ${ }^{116-118}$; see also applications in a extensive review on DFT-based SH. ${ }^{119}$ The main problem of TD-DFT is that the results might depend on the choice of the exchange-correlation functional and in particular on the amount of non-local orbital exchange. Correlated wave-function-based single-reference methods offer a promising alternative. In particular, the algebraic diagrammatic construction method $\operatorname{ADC}(2)^{120,121}$ can produce accurate results at reasonable cost. A number of $\mathrm{ADC}(2)$ dynamics simulations of IC have been reported, ${ }^{122,123}$ and an extension to ISC has been achieved recently. ${ }^{124}$

An additional option, which combines positive aspects of both single- and multi-reference methods, is provided by the spin-flip paradigm. ${ }^{125}$ It has been shown that spin-flip methods do indeed show favorable properties, i.e., a correct topology of conical intersections between 
ground and excited states. ${ }^{126}$ However, dynamics simulations using spin-flip methods have not been carried out so far up to our knowledge.

\subsubsection{Nonadiabatic couplings}

As discussed above, the central quantity for computing the interconversion between states of the same multiplicity is the NAC vector. Given the electronic wave functions of two states $\Psi_{\alpha}$ and $\Psi_{\beta}$, the NAC vector is defined as

$$
\vec{T}_{\beta \alpha}(\vec{R})=\left\langle\Psi_{\beta}(\vec{R})|\vec{\nabla}| \Psi_{\alpha}(\vec{R})\right\rangle
$$

where $\vec{\nabla}$ is the differential operator with respect to the nuclear coordinates $\vec{R}$. The coupling vector measures how much the change in the wave function of one state $\left(\vec{\nabla} \Psi_{\alpha}\right)$ resembles the wave function of a different state $\left(\Psi_{\beta}\right)$. It is large whenever two wave functions exchange their character, which is usually the case around avoided crossings or conical intersections. The NAC vector can be computed using response theory, requiring a similar, albeit somewhat more involved, formalism as the computation of energy gradients. This has been realized for several electronic structure methods, e.g., CASSCF, ${ }^{127,128} \mathrm{MRCI},{ }^{129}$ and TD-DFT, ${ }^{130}$ but is not as readily available as excited-state energies and gradients. In SHARC, NAC vectors are only available from MoLPRo and COLUMBUs, for the CASSCF and MRCI methods.

One caveat of computing NAC vectors for SH simulations is that on the order of $n_{\text {states }}^{2}$ vectors need to be computed every time step, adding considerable cost. Therefore, an alternative method for computing nonadiabatic interactions has been developed following the initial work of Hammes-Schiffer and Tully. ${ }^{70}$ For the application of this method, it is important to realize that for evaluating the EOM not the whole NAC vector is needed but only its projection on the nuclear velocity, i.e., $\vec{T}_{\beta \alpha}(\vec{R}) \cdot \frac{\Delta \vec{R}}{\Delta t}$. It can further be realized that this expression can be discretized according to ${ }^{131}$

$$
\vec{T}_{\beta \alpha}(\vec{R}) \cdot \frac{\Delta \vec{R}}{\Delta t} \approx \frac{1}{\Delta t}\left\langle\Psi_{\beta}(\vec{R}) \mid \Psi_{\alpha}(\vec{R}+\Delta \vec{R})\right\rangle .
$$

The central quantity in this context is the overlap between the wave functions computed at the geometries of subsequent time steps.

The wave function overlap can be computed from the overlaps of the atomic orbitals at the two geometries, the molecular orbital coefficients, as well as the response or CI coefficients. ${ }^{131-133}$ For large wave function expansions, it can become prohibitively expensive to compute this term, primarily because the two molecular orbital sets are non-orthogonal. However, the cost can be drastically reduced by using an optimized algorithm, such as the one proposed in Ref. 131. In this algorithm, one takes advantage of two facts: that the overlap can be factored into independent alpha and beta contributions and that many Slater determinants only differ by one electron. This allows reusing many intermediates in the computation, significantly reducing the scaling of the overlap computation.

The advantage of using wave function overlaps in nonadiabatic dynamics simulations is that they can be readily adapted to various electronic structure methods and quantum chemistry programs. SHARC can currently compute wave function overlaps for all interfaced 
methods-CASSCF, MRCI, ${ }^{131}$ MS-CASPT2, ${ }^{134,135}$ TD-DFT, ${ }^{31}$ and ADC(2). ${ }^{124}$ Furthermore, in situations where the NAC vectors become locally very large and narrow ${ }^{136}$-and thus difficult to integrate numerically-wave function overlaps allow for a more stable propagation with longer time steps, especially when used in connection with the local diabatization method. ${ }^{81}$

\subsubsection{Spin-orbit coupling and scalar relativistic effects}

In general, states of different multiplicity or different spin-magnetic quantum number $M_{S}$ interact due to relativistic effects. The theory of relativistic quantum chemistry, which describes these effects, is exceedingly complex due to both formal and practical issues. ${ }^{34}$ However, some very practical approximations have emerged over the years, which allow including relativistic effects in standard quantum chemistry codes with only little additional effort.

The SOC terms considered here can be defined as the matrix elements of the Breit-Pauli spin-orbit Hamiltonian: ${ }^{34}$

$$
\hat{H}^{\mathrm{SO}, \mathrm{BP}}=\frac{1}{2 c^{2}} \sum_{i}^{n_{\mathrm{el}}} \sum_{A}^{n_{\mathrm{nuc}}} \frac{Z_{A}\left(\vec{r}_{i A} \times \vec{p}_{i}\right) \cdot \vec{s}_{i}}{r_{i A}^{3}}-\frac{1}{2 c^{2}} \sum_{i \neq j}^{n_{\mathrm{el}}} \frac{\left(\vec{r}_{i j} \times \vec{p}_{i}\right) \cdot \vec{s}_{i}}{r_{i j}^{3}}+\frac{1}{c^{2}} \sum_{i \neq j}^{n_{\mathrm{el}}} \frac{\left(\vec{r}_{i j} \times \vec{p}_{i}\right) \cdot \vec{s}_{j}}{r_{i j}^{3}},
$$

where $c$ is the speed of light, $\vec{r}_{i A}$ is the distance vector between electron $i$ and nucleus $A$, $\vec{r}_{i j}$ is the distance vector between electrons $i$ and $j, \vec{p}$ is a momentum vector, and $\vec{s}$ a spin vector. The three terms of the operator are usually called the one-electron term and the two-electron spin-same and spin-other-orbit contributions (from left to right). Physically, the first term describes the interaction of the magnetic moment induced by the orbital motion of an electron around a nucleus with the intrinsic spin magnetic moment, and analogously for the two-electron terms. This effect can couple an $\alpha$ electron in some orbital with a $\beta$ orbital in an orbital with an appropriate different angular momentum, thus introducing a spin-flipping mechanism which is necessary for ISC.

Generally, calculations involving $\hat{H}^{\mathrm{SO}, \mathrm{BP}}$ will be more demanding compared to calculations considering only the $\mathrm{MCH}$, due to the larger number of two-electron integrals needed. Hence, the SOC terms are usually computed in a mean-field approach using either atomic mean-field integrals (AMFI), ${ }^{137,138}$ or the somewhat more sophisticated spin-orbit mean-field (SOMF) approach. ${ }^{139,140}$ In both approaches, the formally two-electron spin-orbit operator is approximated by an effective one-electron operator (reminiscent to Hartree-Fock theory), leading to considerably cheaper computations with little error. ${ }^{137-140}$ In order to arrive at spin-orbit matrix elements between two electronic states, one finally combines the integrals over the effective one-electron operator and the basis functions with the appropriate one-electron spin-transition density matrix. ${ }^{141}$ SHARC currently supports the computation of SOC terms, using either the AMFI or SOMF approaches, for the CASSCF, ${ }^{138} \mathrm{MS}-\mathrm{CASPT} 2,{ }^{138} \mathrm{MRCI},{ }^{142}$ TD-DFT, ${ }^{31,75}$ and $\mathrm{ADC}(2)^{124}$ methods.

Besides SOC, oftentimes scalar relativistic effects are considered. These effects are due to the fact that in heavy atoms the inner shell electrons move at relativistic velocities, which leads to a relativistic mass increase that affects the shapes and energies of the orbitals. Typically, the innermost shells of $s$ type will shrink considerably due to this mass increase, whereas outer shells will expand because the inner shells better shield the nuclear charge. ${ }^{143}$ Scalar 
relativistic effects can be included in electronic structure calculation by means of methods like the zero-order regular approximation ${ }^{144}$ or the Douglas-Kroll-Hess transformation. ${ }^{145}$ Practically, these methods add little extra effort to the calculations, because they mainly affect how the one-electron kinetic energy integrals are computed. Consequently, most electronic structure packages interfaced to SHARC can compute scalar relativistic effects, which are routinely included in SHARC simulations.

\subsubsection{Dipole moments and Dyson norms}

In order to incorporate DFCs into the SH simulation, it is also necessary to compute the static dipole moments of the electronic states as well as the transition dipole moments between them. This gives rise to a matrix of dipole moment vectors:

$$
\vec{\mu}_{\beta \alpha}=\left\langle\Psi_{\beta}\left|-\sum_{i}^{n_{\mathrm{el}}} \vec{r}_{i}+\sum_{A}^{n_{\mathrm{nuc}}} Z_{A} \vec{R}_{A}\right| \Psi_{\alpha}\right\rangle .
$$

These terms are readily available for methods producing explicit wave functions such as CASSCF and MRCI. In these cases, the terms are simply computed as operator expectation values and matrix elements between the different wave functions. ${ }^{146}$ In the case of (singlereference) response methods, such as TD-DFT and CC2, the situation is more involved. While the matrix elements involving the ground state $\left(\mu_{00}\right.$ and $\left.\mu_{0 \alpha}\right)$ are usually easily computed, the excited-state (transition) dipole moments $\left(\mu_{\beta \beta}\right.$ and $\left.\mu_{\beta \alpha}\right)$ require the solution of additional response equations, ${ }^{147}$ or might not be available at all.

Another related quantity is the photoionization probability, which is needed if the interaction with an electric field leads to ionization. These probabilities might be approximated by means of the norms of Dyson orbitals, ${ }^{148,149}$ which are relatively cheap to calculate and can thus be used on-the-fly. The Dyson orbital between an $n$-electron wave function $\Psi_{\beta}$ and an ionized $(n-1)$-electron wave function $\Theta_{\alpha}$ is defined as

$$
\phi_{\beta \alpha}^{\text {Dyson }}(\vec{r})=\int \ldots \int \Psi_{\beta}\left(\vec{r}, \vec{r}_{2}, \ldots, \vec{r}_{n}\right) \Theta_{\alpha}\left(\vec{r}_{2}, \ldots, \vec{r}_{n}\right) \mathrm{d} \vec{r}_{2} \ldots \mathrm{d} \vec{r}_{n} .
$$

This equation is closely related to the one for wave function overlaps, described in section 10.4.2, with the exception that one electron is "left out". The practical implementation therefore involves a loop over multiple overlap calculations, one for each occupied orbital. ${ }^{150}$ Dyson norms are currently available in SHARC for CASSCF, MS-CASPT2, MRCI, and TD-DFT methods.

\subsection{Analysis}

Nonadiabatic dynamics simulations produce a wealth of information for each time step: nuclear positions and velocities, electronic populations and energies, information on the active state, hopping probabilities, etc. In order to extract chemical meaning and to make useful predictions ${ }^{151}$ data analysis is necessary.

In general, one can distinguish between (i) predicted values for physical observables and (ii) non-observable descriptors. ${ }^{60,151}$ The former quantities can be directly related to data measured 
experimentally and are therefore also valuable to estimate the accuracy of the simulations. Non-observable descriptors, i.e., quantities that are not directly accessible by experiments, for example wave functions, can facilitate discussions of reaction mechanisms, enable generalizations to whole classes of molecules, and allow comparison to other computational simulations, i.e., provide additional insight that is not necessarily accessible experimentally.

\subsubsection{Simulation of observables}

Among the basic observables which can be simulated by nonadiabatic dynamics methods are different types of product branching ratios. ${ }^{60}$ A prime example of this are quantum yields, either related to different electronic states-e.g., the electronic ground state, long-lived triplet states, or ionic states-or to different stable molecular arrangements-e.g., in photoinduced rearrangement reactions. Quantum yields of electronic states can easily be computed from the electronic populations-i.e., the absolute squares of the coefficients $\left|c_{\alpha}(t)\right|^{2}$ summed over all trajectories-or from the fraction of trajectories moving in a particular state at the end of the simulation. Furthermore, for dissociation or scattering reactions, kinetic energy distributions and velocity correlations ${ }^{60,152}$ can be directly extracted from the trajectories.

[Figure 3 about here.]

Another important class of observables are transient signals measured in pump-probe spectroscopic experiments. The basis principles behind some popular time-resolved methods and their corresponding signals are sketched in Figure 3. One of the most ubiquitous pumpprobe techniques for gas-phase studies is time-resolved photoelectron spectroscopy (TRPES). ${ }^{10}$ Within this method, after the pump pulse, the excited molecule is probed with a probe laser pulse which is capable of ionizing the molecule, see Figure 3a. This is accomplished either through high photon energies (leading to single-photon ionization) or through large intensities (inducing two- or multiphoton ionization). The ejected electron and the remaining molecular ion can then be detected through mass spectrometric techniques. In TRPES, only the number and energy of ejected electrons are measured depending on the pump-probe delay. A computational simulation of a TRPES signal therefore requires that for each time step and trajectory one can compute the probability of ejecting an electron from the current active electronic state, given the laser characteristics. The simplest way to do that is to approximate the ionization probabilities by means of Dyson norms, which describe the compatibility between a neutral and an ionic wave function (see above). ${ }^{15}$ This means that in addition to the neutral states calculated during the dynamics simulation, a number of ionic states are also necessary for each time step. In turn, this might affect the choice of electronic structure method because it needs to describe the neutral and ionic states in a balanced way.

Ionization probabilities can be computed by more elaborate methods beyond Dyson norms, taking into account the wave function of the outgoing electron and the coupling between different ionization channels. ${ }^{148,153-155}$ Either way, the ionization probability data from each trajectory and time step can be combined to obtain a time-dependent signal

$$
\sigma^{\mathrm{TRPES}}(t, E)=\sum_{a}^{n_{\text {traj }}} \sum_{\alpha}^{n_{\text {state }}} I_{\beta \rightarrow \alpha, a}\left(t, E, E_{\text {photon }}, E_{\alpha}(t)-E_{\beta}(t)\right),
$$


where $\beta$ is the active state at the respective time step. When using Dyson norms, the equation can be stated as

$$
\sigma^{\mathrm{TRPES}}(t, E)=\sum_{a}^{n_{\text {traj }}} \sum_{\alpha}^{n_{\text {state }}}\left|\phi_{\beta \alpha, a}^{\text {Dyson }}(t)\right|^{2} \exp \left(-\frac{\left(E-E_{\text {photon }}-E_{\beta, a}(t)+E_{\alpha, a}(t)\right)^{2}}{2 w^{2}}\right),
$$

where $w$ is the width parameter of the employed Gaussian broadening. The quantity $\sigma^{\operatorname{TRPES}}(t, E)$ can then be convoluted with the respective instrument response function to arrive at the final simulated TRPES signal. ${ }^{15,156,157}$

There also exist a number of more elaborate experimental methods related to TRPES ${ }^{6}-$ like time-resolved photoelectron photoion coincidence detection, velocity map imaging, or COLTRIMS (cold target recoil ion momentum spectrometer)-which also measure the momentum of the photoelectron, as well as number, energies, and momenta of the photoion, but to our knowledge corresponding on-the-fly simulations were not attempted to-date.

Another popular pump-probe technique-mostly for solvent-phase experiments-is transient absorption spectroscopy (TAS). ${ }^{12}$ Here, the excited molecule is probed by the absorption of a broad-band pulse in the UV or visible range, see Figure 3b. Because the absorption depends on the populated electronic state, the excited-state dynamics can be followed by changes in the resulting time-dependent absorption spectrum. The simulation of such a spectrum is similar to the case of TRPES signals, involving equations analogous to Eq. (20). Instead of computing ionic states, it is necessary to compute additional, higher-energy neutral states, such that excitations from the current active state are possible. The absorption probabilities are here given by the transition dipole moments between the currently active state and the higher states. If the transition dipole moments to the lower states are also included, it is possible to obtain stimulated emission signals in the TAS, too. More elaborate methods for computing TAS signals from $\mathrm{SH}$ trajectories also exist. ${ }^{158}$

Yet another pump-probe technique to detect excited-state dynamics is time-resolved infrared spectroscopy (TRIR). ${ }^{159}$ This method probes the time-dependent oscillations of the molecule and its permanent dipole moment after excitation (see Figure 3c). In order to simulate a TRIR spectrum from a trajectory, it is necessary to compute the permanent dipole moment of the active state for all time steps, a task which is automatically done in SHARC. Then, the autocorrelation function of the dipole moment vectors can be computed. A Fourier transformation of this function would yield the static IR spectrum of the molecule, ${ }^{160}$ however, no timedependent information is retained. Instead, a time-frequency distribution function can be computed, e.g., from short-time Fourier transformations or wavelet transformations. Unlike TRPES and TAS above, the simulation of TRIR spectra from a trajectory does not require the computation of additional electronic states.

\subsubsection{Analysis of electronic evolution}

While the output of all pump-probe experiments is some kind of transient signal, most often one is interested in the temporal evolution of the excited-state populations. Signals and populations are clearly related, but often in a convoluted way because processes and signals can overlap and exhibit additional dependencies. In contrast, excited-state simulations directly provide excited-state populations, albeit in a particular representation, recall Figure 1. 
There are different ways to compute populations in SH simulations. One can track the fraction of trajectories in each state (giving the "classical" populations) or compute the sum of the amplitudes $\left|c_{\alpha}(t)\right|^{2}$ over all trajectories (the "quantum" populations). If the simulations correctly included decoherence (see section 10.3.2), then these two quantities will be consistent and give the populations in terms of the actually used representation. In SHARC, this will be the diagonal representation, but it is also possible to transform the populations back into the $\mathrm{MCH}$ representation, with the states as produced by the quantum chemistry calculation $\left(S_{0}, S_{1}, T_{1}, \ldots\right)$. These populations can subsequently be fitted-either with simple exponential functions, or with more extensive kinetic models ${ }^{26}$-to obtain time constants that can be compared to experiment.

Whereas the picture of adiabatic states is well defined from a computational point of view, it is not generally the most meaningful way to discuss the physical processes occurring. Interpretation is facilitated by transforming the populations into the spectroscopic representation, yielding populations for the different state characters, e.g. ${ }^{1} n \pi^{*},{ }^{1} \pi \pi^{*},{ }^{3} n \pi^{*},{ }^{3} \pi \pi^{*}$. No general and rigorous strategy exists to perform this transformation, but a number of approximate strategies exist: (i) computing a path-integral of the overlap or NAC along a trajectory, (ii) diabatization using physical observables, and (iii) diabatization using electronic structure analysis methods as described below. Some of us used strategy (i) for the simulation of ISC in $\mathrm{SO}_{2},{ }^{28}$ strategy (ii) for the study of the dynamics of the keto and enol tautomers of cytosine, ${ }^{89}$ and strategy (iii) in model systems to investigate charge ${ }^{161}$ and energy transfer. ${ }^{136}$

A central problem in the adiabatic (i.e., $\mathrm{MCH}$ )-to-diabatic transformation is the identification of the dominant state character of the current $\mathrm{MCH}$ wave functions. The most straightforward way is to analyze the molecular orbitals (MOs)-i.e., the highest occupied MO (HOMO), the lowest unoccupied MO (LUMO), the LUMO+1, etc.-and the excited-state response vector to find out which orbitals are involved in the transition. This method works in simple cases but has two severe downsides. First, the state character identification can be ambiguous if the orbitals are mixed or several configurations are involved, as it is not possible to simply add up all contributions-any interference terms have to be included in a consistent manner. ${ }^{162}$ Second, a manual inspection of the involved orbitals is unfeasible if a large number of computations is performed, as is the case in on-the-fly dynamics simulations. The application of some well-established visualization techniques such as the natural transition orbitals ${ }^{163}$ or the attachment-detachment densities ${ }^{164}$ can be used to solve the first problem. ${ }^{165}$

Solving the second problem is a more involved task, as it requires a completely automated analysis of excited state character. To this end, a set of analysis strategies based on the one-electron transition density matrix was developed within the framework of exciton theory. ${ }^{165-167}$ A fundamental concept in this formalism is the computation of charge transfer numbers (see, e.g., Ref. 168). These allow to partition the electronic excitation into individual local and charge transfer contributions. These methods have been previously applied in static computations to study a number of systems, such as DNA ${ }^{169-171}$ or conjugated polymers. ${ }^{172}$ It could also be used to automatically distinguish between different classes of states in metal complexes, e.g., metal-centered states, metal-to-ligand charge transfer states, or intraligand states. ${ }^{173}$ The possibility of employing this automated state identification in dynamics simulations was outlined in the case of monitoring energy transfer in the pyridone dimer. ${ }^{136}$ 


\subsubsection{Analysis of nuclear evolution}

Besides the analysis of the electronic degrees of freedom, it is also very important to evaluate the evolution of the molecular geometry, which can undergo drastic changes during the excited-state dynamics. A convenient way to monitor these changes is in terms of internal coordinates, for example, bond distances, angles, dihedrals, or out-of-plane motions. Also of relevance in many cases are ring conformation parameters, ${ }^{174,175}$ or other large-scale motions, which can be tracked, e.g., with intermolecular distances. This approach was applied, e.g., to monitor ring deformation in nucleobases ${ }^{176}$ and analogues ${ }^{26}$ or to follow excited-state proton transfer processes. ${ }^{177}$ Furthermore, internal coordinate evolution can also be a proxy to observe the electronic populations, if different state characters exhibit significantly different equilibrium values for internal modes. ${ }^{26}$ Experimentally, using the emerging techniques of ultrafast X-Ray diffraction ${ }^{178}$ or ultrafast electron diffraction, ${ }^{179}$ it is possible to follow the evolution of internal coordinates through time-resolved (radial) distribution functions.

Besides internal coordinate analysis, normal mode analysis protocols for molecular dynamics simulations were developed, ${ }^{180,181}$ in light of the fact that coherent normal-mode oscillations are readily observed in modern femtosecond experiments. ${ }^{182}$ The method available in SHARC ${ }^{181}$ proceeds by transforming the Cartesian coordinates of the structures reached during the dynamics simulations into normal mode displacements. The normal mode displacements are in turn subjected to various statistical analysis procedures to obtain the coherent and total normal-mode activity during the dynamics. The method, applied in the case of excited state proton transfer, ${ }^{181}$ can reproduce the experimentally observed ${ }^{183}$ normal-mode activation.

Dynamics simulations can also help to discover stationary points and minimum-energy crossing points. The latter can be found by statistical analysis of the geometries where surface hops occurred-clustering can, for example, unravel the number of involved minimum-energy conical intersections. Similarly, statistical analysis of all geometries can find local minima, because many time steps of the trajectories will be located in the vicinity of these minima. The identification of these stationary points can in turn facilitate the formulation of a general relaxation mechanism. ${ }^{27}$

\subsection{Example application}

In this section, we would like to illustrate some of the theoretical points discussed above with one example. Cytosine is one of the chromophores in DNA and therefore has been an interesting subject of study. ${ }^{91,184-187}$ SHARC has been employed to unravel its excited-state dynamics ${ }^{20,21}$ and simulate its time-resolved photo-electron spectrum. ${ }^{15}$ Our study aimed at the simulation of cytosine in the gas phase, where different tautomers of the molecule are known to coexist. The two most important ones are the amino-keto ("keto cytosine") and amino-enol ("enol cytosine") tautomers. ${ }^{21}$

The first step of the study was the determination of the electronic structure level of theory to use in dynamics. We chose the SA-CASSCF $(12,9) / 6-31 G^{*}$ level of theory, averaging four singlets and three triplets for keto cytosine, and three singlets and four triplets for enol cytosine with equal weights. This state-averaging protocol was able to reproduce the correct 
state ordering (with respect to MS-CASPT2 calculations) of the few lowest $n \pi^{*}$ and $\pi \pi^{*}$ states at the Franck-Condon point (i.e., the ground-state equilibrium geometry), which was optimized for each tautomer beforehand. The active space contained 12 electrons in 9 active orbitals, including the two lone pairs of cytosine, plus the orbitals of the $\pi$ system (minus the $\pi$ of the amino group). This level of theory was chosen as a compromise between computational cost and accuracy, using the largest feasible active space. Furthermore, at that time, CASSCF in MolPro was virtually the only method where excited states including SOCs and NACs (see section 10.4) were available.

The second step was the preparation of the initial conditions, as outlined in section 10.3.4. Cytosine in the ground state is not always exactly at the equilibrium (Franck-Condon) geometry. Instead, it vibrates with the available ZPE and it samples different configurations that need to be considered as initial geometries for the excited-state dynamics. Separately for each tautomer, the sampling of these initial geometries was carried out according to a Wigner distribution which mimics the probability distribution of the geometries and velocities of the vibrational ground-state. In order to compute the Wigner distributions, we performed harmonic frequency calculations of the respective optimized ground-state equilibrium geometries. The Wigner sampling eventually yielded a list of geometries (and corresponding velocities), and for each geometry a vertical-excitation calculation was performed. The calculated excitation energies and oscillator strengths were employed in a probabilistic manner ${ }^{86}$ to determine whether a given geometry would be excited by a delta laser pulse, and which excited state would be populated by the pulse. Consequently, after this probabilistic state selection scheme, we obtained a list of initial conditions-with each one being a combination of geometry, velocities, and initial state-which could be employed as the starting point of one trajectory.

Inspection of initial conditions reveals how important the different electronic state representations are (see Figure 1). Even though the relevant bright state, the first $\pi \pi^{*}$ state, at the Franck-Condon point is the $S_{1}$ state (i.e., the lowest-energy excited singlet), many initial conditions were obtained where the $S_{2}$ was the bright state. The reason is that in keto cytosine, a conical intersection between the $\pi \pi^{*}$ and an $n \pi^{*}$ state is located very close to the Franck-Condon point and therefore a small displacement away from this geometry is sufficient to lead to a reordering of these two states, such that the $\pi \pi^{*}$ state becomes the $S_{2}$. This shows that in the case of keto cytosine, it would be inaccurate to use the labels " $S_{1}$ " or " $S_{2}$ " in order to discuss the bright $\pi \pi^{*}$ state beyond the frozen equilibrium geometry, and that a set of initial conditions that sample the whole Franck-Condon region is necessary.

As a third step, we performed nonadiabatic dynamics simulations and carried out the trajectory analysis for 68 and 65 trajectories in the keto and enol tautomers, respectively. The resulting excited-state populations for the two forms are shown in Fig. 4a and 4b. Although the trajectories were propagated in the diagonal representation internally in the SHARC program, the figure shows the populations in the spectroscopic representation. This was accomplished by classifying the diagonal states as ground state (GS), ${ }^{1} \pi \pi^{*},{ }^{1} n \pi^{*}$, or triplet (T) based on the magnitude of the transition dipole moment between the state of interest and the lowest-energy state. Bright states were classified as ${ }^{1} \pi \pi^{*}$, less bright state as ${ }^{1} n \pi^{*}$, and very dark states as triplet (see Ref. 21 for thresholds).

[Figure 4 about here.] 
[Table 2 about here.]

According to the figure, the initial population is primarily located in the bright ${ }^{1} \pi \pi^{*}$ state (which is a mixture of the $S_{1}$ and $S_{2}$ states) for both keto and enol tautomers and it decays with a time constant of approximately $25 \mathrm{fs}$ ( $40 \mathrm{fs}$ ) for the keto (enol) tautomer. The decaying population is transferred to the ${ }^{1} n \pi^{*}$ state, from where it can subsequently convert to the ground state or undergo ISC to a triplet state. The decay time of this ${ }^{1} n \pi^{*}$ state is a combination of the IC and ISC decay channels, and amounts to $216 \mathrm{fs}$ (1513 fs) for the keto (enol) tautomer. The rise time of the triplet state population was determined to be $745 \mathrm{fs}$ $(6220 \mathrm{fs})$ for the keto (enol) tautomer. The three time constants are given in Table 2. These time constants have been also heavily discussed in experimental investigations, although there the population dynamics can only be inferred indirectly.

As a final, fourth step, we computed TRPES signals, ${ }^{15}$ since it has been largely used to probe the dynamics of cytosine. ${ }^{188-191}$ The goal of TRPES is to follow the ionization probability of the currently occupied (neutral) excited state. Unfortunately, the TRPES signals do not only depend on the how the neutral excited-state populations actually change in time but also on the geometry-dependent ionization probabilities between the electronic states. Therefore, it is not enough to compare the experimental signal with the excited-state population, but one should also simulate the actual probe step. As a crude approximation of the ionization yield, we used Dyson norms (see sections 10.4.4 and 10.5). The resulting simulated ionization yields are shown in Fig. $4 \mathrm{c}$ and $4 \mathrm{~d}$. The signals for keto and enol cytosine are scaled relative to each other and reflect the abundance of approximately 35\% keto and 65\% enol tautomer in the gas phase. ${ }^{192}$ As given in Table 2, the time constants for the ionization signals of the different states is different from the time constants obtained from the population dynamics.

\section{[Figure 5 about here.]}

The experimental photoelectron yield ${ }^{189}$ of gas phase cytosine is shown in Fig. 5. A fit of this yield showed that the curve could be represented as the sum of two (temporally broadened) exponentials (dashed lines). The corresponding time constants were determined to be $820 \mathrm{fs}$ and $3200 \mathrm{fs} .{ }^{189}$ Strikingly enough, although the computations produced quite different time constants, the computed total photoelectron yield from the SHARC simulations (based on CASSCF potentials and Dyson norms with their inherent limitations) agrees well with the experimental photoelectron yield, as shown in Fig. 5. The comparison of these numbers with the outcome of the simulations teaches that the experimental signal is the sum of different underlying contributions and that this sum cannot easily be decomposed into those contributions without additional information. ${ }^{15}$ This is why a collaborative effort between experiment and theory is of utmost importance to gain a deeper understanding of the complex interplay between electrons, nuclei and electromagnetic fields.

\subsection{Summary}

The simulation of light-induced processes, where the motion of electrons and nuclei are correlated, poses many challenges. ${ }^{193}$ In this chapter, we describe a general surface-hopping method able to follow the ultrafast nonadiabatic dynamics of molecular systems. This method, 
abbreviated SHARC (Surface Hopping including ARbitrary Couplings), allows for the simulation of molecular systems with dozens of atoms including all degrees of freedom and any type of coupling. As such, SHARC is able to simulate the direct interaction of the molecule with explicit laser pulses as well as to describe internal conversion and intersystem crossing. As a mixed quantum-classical method, where the nuclei are treated classically and only the electrons are described quantum mechanically, SHARC cannot take into account quantum phenomena, such as tunnelling, quantum interferences, or (de)-coherences. However, SHARC and in general surface-hopping methods have demonstrated to be enormously successful in the study of nonadiabatic phenomena for different neutral molecular system in gas phase, from small organic/inorganic chromophores or medium size transition metal complexes. The implementation of hybrid quantum mechanical/molecular mechanical (QM/MM) approaches to describe nonadiabatic dynamics in the presence of an environment is in progress and it will see large applicability in the realm of photobiology and photochemistry in solution.

Trajectories provide the time evolution of state populations, where the character of the associated wave functions, and the conformational changes can be resolved. The analysis of all underlying data can reveal reaction mechanisms, as well as help in the determination of time scales and quantum yields. A direct comparison of calculated reaction mechanism and time-resolved populations with experimental observables is difficult, since time-resolved experiments can only infer neutral dynamics indirectly. Alternatively, SHARC can also simulate the experimental probe process, e.g., ionization, to allow for a direct comparison to the experimental observable. Ionization can be approximated via the simple approach of calculating Dyson norms but more sophisticated methods, e.g., Coulomb waves, ${ }^{155}$ discretization of the ionic continuum, ${ }^{194}$ B-splines, ${ }^{195}$ Stieltjes imaging, ${ }^{196}$ or time-dependent resolution in ionic states (TD-RIS) ${ }^{149,153}$ might be needed to obtain a better description of the ejected ion and a better comparison to time-resolved photoelectron spectra. In SHARC it is even possible to simulate the interaction of molecules with strong fields. However, in this case it is essential to get very accurate excited-state energies and transition dipole moments for a large number of high-lying electronic states. This is because the Stark shifts induced by the strong fields are very sensitive to tiny inaccuracies in the state energies and the transition dipole moments. ${ }^{197}$ In these cases, it might be useful to employ simpler models with fitted effective parameters that allow multiphoton processes, ${ }^{198,199}$ but to allow for the full dimensionality of the electronic and nuclear motion. In the realm of attochemistry, a more detailed analysis of the electronic wave function and the tracking of its evolution ${ }^{14}$ could open new possibilities to the field of surface hopping.

\section{Acknowledgment}

We thank the Austrian Science Fund (FWF) within project I2883 (DeNeTheor) and the University of Vienna for financial support. The authors also would like to thank the past members of the SHARC development team (Martin Richter, Matthias Ruckenbauer, Markus Oppel, and Andrew J. Atkins), as well as the COST actions CM1405 (MOLIM) and CM1305 (ECOSTBio) for fruitful discussions about nonadiabatic dynamics of spin-change processes. The Vienna Scientific Cluster (VSC) is acknowledged for the generous allocation of computer resources which made possible several large-scale SHARC simulations. 


\section{References}

1. P. Agostini and L. F. DiMauro, Rep. Prog. Phys., 2004, 67, 813.

2. M. F. Kling and M. J. Vrakking, Annu. Rev. Phys. Chem., 2008, 59, 463-492.

3. F. Krausz and M. Ivanov, Rev. Mod. Phys., 2009, 81, 163-234.

4. W. Li, A. A. Jaroń-Becker, C. W. Hogle, V. Sharma, X. Zhou, A. Becker, H. C. Kapteyn and M. M. Murnane, Proc. Natl. Acad. Sci., 2010, 107, 20219-20222.

5. F. Calegari, G. Sansone, S. Stagira, C. Vozzi and M. Nisoli, f. Phys. B: At., Mol. Opt. Phys., 2016, 49, 062001.

6. M. Nisoli, P. Decleva, F. Calegari, A. Palacios and F. Martín, Chem. Rev., 2017, 117, 10760-10825.

7. A. H. Zewail, Angew. Chem. Int. Ed., 2000, 39, 2586-2631.

8. M. Dantus and A. H. Zewail (Eds.), Chem. Rev., 2004, 104, 1717-2124.

9. A. H. Zewail, Annu. Rev. Phys. Chem., 2006, 57, 65-103.

10. A. Stolow, A. E. Bragg and D. M. Neumark, Chem. Rev., 2004, 104, 1719-1758.

11. T. Seideman, Annu. Rev. Phys. Chem., 2002, 53, 41-65.

12. R. Berera, R. Grondelle and J. T. M. Kennis, Photosynth. Res., 2009, 101, 105-118.

13. W. Li, X. Zhou, R. Lock, S. Patchkovskii, A. Stolow, H. C. Kapteyn and M. M. Murnane, Science, 2008, 322, 1207-1211.

14. F. Calegari, D. Ayuso, A. Trabattoni, L. Belshaw, S. De Camillis, S. Anumula, F. Frassetto, L. Poletto, A. Palacios, P. Decleva, J. B. Greenwood, F. Martín and M. Nisoli, Science, 2014, 346, 336-339.

15. M. Ruckenbauer, S. Mai, P. Marquetand and L. González, Sci. Rep., 2016, 6, 35522.

16. M. Richter, P. Marquetand, J. González-Vázquez, I. Sola and L. González, f. Chem. Theory Comput., 2011, 7, 1253-1258.

17. S. Mai, P. Marquetand and L. González, Int. J. Quantum Chem., 2015, 115, 1215-1231.

18. S. Mai, M. Richter, M. Ruckenbauer, M. Oppel, P. Marquetand and L. González, SHARC: Surface Hopping Including Arbitrary Couplings - Program Package for Non-Adiabatic Dynamics, sharc-md.org, 2014.

19. M. Barbatti, WIREs Comput. Mol. Sci., 2011, 1, 620-633.

20. M. Richter, P. Marquetand, J. González-Vázquez, I. Sola and L. González, J. Phys. Chem. Lett., 2012, 3, 3090-3095.

21. S. Mai, P. Marquetand, M. Richter, J. González-Vázquez and L. González, ChemPhysChem, 2013, 14, 2920-2931.

22. M. Richter, S. Mai, P. Marquetand and L. González, Phys. Chem. Chem. Phys., 2014, 16, 24423-24436.

23. S. Mai, M. Richter, P. Marquetand and L. González, Ultrafast Phenomena XIX, Springer International Publishing, 2015, vol. 162, pp. 509-513.

24. S. Mai, M. Richter, P. Marquetand and L. González, Chem. Phys., 2017, 482, 9-15.

25. C. E. Crespo-Hernández, L. Martínez-Fernández, C. Rauer, C. Reichardt, S. Mai, M. Pollum, P. Marquetand, L. González and I. Corral, J. Am. Chem. Soc., 2015, 137, 4368-4381. 
26. S. Mai, P. Marquetand and L. González, J. Phys. Chem. Lett., 2016, 7, 1978-1983.

27. S. Mai, M. Pollum, L. Martínez-Fernández, N. Dunn, P. Marquetand, I. Corral, C. E. Crespo-Hernández and L. González, Nat. Commun., 2016, 7, 13077.

28. S. Mai, P. Marquetand and L. González, f. Chem. Phys., 2014, 140, 204302.

29. M. Marazzi, S. Mai, D. Roca-Sanjuán, M. G. Delcey, R. Lindh, L. González and A. Monari, f. Phys. Chem. Lett., 2016, 7, 622-626.

30. C. Rauer, J. J. Nogueira, P. Marquetand and L. González, f. Am. Chem. Soc., 2016, 138, 15911-15916.

31. A. J. Atkins and L. González, f. Phys. Chem. Lett., 2017, 8, 3840-3845.

32. J. Cao, Z.-Z. Xie and X. Yu, Chem. Phys., 2016, 474, 25 - 35.

33. K. G. Dyall and K. Fægri, Introduction to Relativistic Quantum Chemistry, Oxford University Press, 2007.

34. M. Reiher and A. Wolf, Relativistic Quantum Chemistry, Wiley VCH Verlag Weinheim, 2009.

35. B. K. Kendrick, C. A. Mead and D. G. Truhlar, Chem. Phys. Lett., 2000, 330, 629 - 632.

36. M. Baer, Chem. Phys. Lett., 2000, 330, $633-634$.

37. C. M. Marian, WIREs Comput. Mol. Sci., 2012, 2, 187-203.

38. J. N. Harvey, S. Grimme, M. Woeller, S. D. Peyerimhoff, D. Danovich and S. Shaik, Chem. Phys. Lett., 2000, 322, 358 - 362.

39. R. Englman and J. Jortner, Mol. Phys., 1970, 18, 145-164.

40. L. J. Butler, Annu. Rev. Phys. Chem., 1998, 49, 125.

41. M. Born and R. Oppenheimer, Ann. Phys., 1927, 389, 457-484.

42. N. L. Doltsinis, Computational Nanoscience: Do It Yourself!, John von Neuman Institut for Computing, Jülich, 2006, vol. 31, pp. 389-409.

43. E. Schrödinger, Phys. Rev., 1926, 28, 1049-1070.

44. J. S. Briggs and J. M. Rost, Foundation of Physics, 2001, 31, 693-712.

45. H.-D. Meyer, F. Gatti and G. A. Worth, Multidimensional Quantum Dynamics, Wiley-VCH Verlag GmbH \& Co. KGaA, 2009.

46. Q. Meng and H.-D. Meyer, F. Chem. Phys., 2013, 138, 014313.

47. J. C. Tully, f. Chem. Phys., 1990, 93, 1061-1071.

48. N. Balakrishnan, C. Kalyanaraman and N. Sathyamurthy, Phys. Rep., 1997, 280, 79-144.

49. W. H. Miller, f. Phys. Chem. A, 2001, 105, 2942-2955.

50. M. Ben-Nun and T. J. Martínez, Advances in Chemical Physics, John Wiley \& Sons, Inc., 2002, vol. 121, pp. 439-512.

51. X. Li, J. C. Tully, H. B. Schlegel and M. J. Frisch, F. Chem. Phys., 2005, 123, 084106.

52. D. J. Tannor, Introduction to Quantum Mechanics: A Time-Dependent Perspective, University Science Books, 2006.

53. A. Abedi, N. T. Maitra and E. K. U. Gross, Phys. Rev. Lett., 2010, 105, 123002.

54. S. Habershon, D. E. Manolopoulos, T. E. Markland and T. F. M. III, Annu. Rev. Phys. Chem., 2013, 64, 387-413. 
55. G. Richings, I. Polyak, K. Spinlove, G. Worth, I. Burghardt and B. Lasorne, Int. Rev. Phys. Chem., 2015, 34, 269-308.

56. B. F. E. Curchod, C. Rauer, P. Marquetand, L. González and T. J. Martínez, f. Chem. Phys., 2016, 144, 101102.

57. J. E. Subotnik, A. Jain, B. Landry, A. Petit, W. Ouyang and N. Bellonzi, Annu. Rev. Phys. Chem., 2016, 67, 387-417.

58. A. Warshel and M. Karplus, Chem. Phys. Lett., 1975, 32, 11 - 17.

59. J. C. Tully and R. K. Preston, f. Chem. Phys., 1971, 55, 562-572.

60. M. Persico and G. Granucci, Theor. Chem. Acc., 2014, 133, 1526.

61. S. C. Cheng, C. Zhu, K. K. Liang, S. H. Lin and D. G. Truhlar, f. Chem. Phys., 2008, 129, 024112.

62. G. Granucci, M. Persico and A. Zoccante, f. Chem. Phys., 2010, 133, 134111.

63. J. E. Subotnik and N. Shenvi, f. Chem. Phys., 2011, 134, 024105.

64. M. Ben-Nun and T. J. Martínez, f. Chem. Phys., 1998, 108, 7244-7257.

65. D. V. Makhov, W. J. Glover, T. J. Martinez and D. V. Shalashilin, J. Chem. Phys., 2014, 141, 054110.

66. U. Manthe and H. Köppel, f. Chem. Phys., 1990, 93, 1658-1669.

67. W. Domcke and G. Stock, in Theory of Ultrafast Nonadiabatic Excited-State Processes and their Spectroscopic Detection in Real Time, ed. I. Prigogine and S. A. Rice, John Wiley \& Sons, Inc., 2007, vol. 100, ch. 1, pp. 1-169.

68. G. Stock and U. Müller, f. Chem. Phys., 1999, 111, 65-76.

69. M. Barbatti and K. Sen, Int. F. Quantum Chem., 2016, 116, 762-771.

70. S. Hammes-Schiffer and J. C. Tully, f. Chem. Phys., 1994, 101, 4657-4667.

71. F. A. Shakib and P. Huo, f. Phys. Chem. Lett., 2017, 8, 3073-3080.

72. T. Fleig, Chem. Phys., 2012, 395, 2 - 15.

73. V. Vallet, L. Maron, C. Teichteil and J.-P. Flament, f. Chem. Phys., 2000, 113, 1391-1402.

74. R. Winkler, in Spin-Orbit Coupling Effects in Two-Dimensional Electron and Hole Systems, Springer Berlin Heidelberg, Berlin, Heidelberg, 2003, ch. Quasi-Degenerate Perturbation Theory, pp. 201-206.

75. F. Wang and T. Ziegler, f. Chem. Phys., 2005, 123, 154102.

76. P. Marquetand, M. Richter, J. González-Vázquez, I. Sola and L. González, Faraday Discuss., 2011, 153, 261-273.

77. M. F. Herman, f. Chem. Phys., 1999, 111, 10427-10435.

78. G. Granucci, M. Persico and G. Spighi, f. Chem. Phys., 2012, 137, 22 A501.

79. P. J. Kuntz, f. Chem. Phys., 1991, 95, 141-155.

80. M. Pederzoli and J. Pittner, f. Chem. Phys., 2017, 146, 114101.

81. G. Granucci, M. Persico and A. Toniolo, F. Chem. Phys., 2001, 114, 10608-10615.

82. N. Klaffki, O. Weingart, M. Garavelli and E. Spohr, Phys. Chem. Chem. Phys., 2012, 14, 14299-14305. 
83. R. Schinke, Photodissociation Dynamics: Spectroscopy and Fragmentation of Small Polyatomic Molecules, Cambridge University Press, 1995.

84. J. P. Dahl and M. Springborg, f. Chem. Phys., 1988, 88, 4535-4547.

85. M. Garavelli, F. Bernardi, M. Olivucci, M. J. Bearpark, S. Klein and M. A. Robb, F. Phys. Chem. A, 2001, 105, 11496-11504.

86. M. Barbatti, G. Granucci, M. Persico, M. Ruckenbauer, M. Vazdar, M. Eckert-Maksić and H. Lischka, F. Photochem. Photobiol. A, 2007, 190, 228-240.

87. P. G. Szalay, T. Müller, G. Gidofalvi, H. Lischka and R. Shepard, Chem. Rev., 2012, 112, 108-181.

88. B. O. Roos, P. R. Taylor and P. E. M. Siegbahn, Chem. Phys., 1980, 48, 157-173.

89. S. Mai, P. Marquetand, M. Richter, J. González-Vázquez and L. González, ChemPhysChem, 2013, 14, 2920-2931.

90. F. M. Siouri, S. Boldissar, J. A. Berenbeim and M. S. de Vries, f. Phys. Chem. A, 2017, 121, 5257-5266.

91. S. Mai, M. Richter, P. Marquetand and L. González, Photoinduced Phenomena in Nucleic Acids I, Springer Berlin Heidelberg, 2015, vol. 355, pp. 99-153.

92. M. Assmann, T. Weinacht and S. Matsika, F. Chem. Phys., 2016, 144, 034301.

93. T. J. Martínez, Acc. Chem. Res., 2006, 39, 119-126.

94. D. Polli, P. Altoe, O. Weingart, K. M. Spillane, C. Manzoni, D. Brida, G. Tomasello, G. Orlandi, P. Kukura, R. A. Mathies, M. Garavelli and G. Cerullo, Nature, 2010, 467, 440-443.

95. G. Cui and W. Fang, f. Phys. Chem. A, 2011, 115, 11544-11550.

96. M. Barbatti, M. Vazdar, A. J. A. Aquino, M. Eckert-Maksić and H. Lischka, f. Chem. Phys., $2006,125,164323$.

97. M. Richter and B. P. Fingerhut, f. Chem. Theory Comput., 2016, 12, 3284-3294.

98. A. Banerjee, D. Halder, G. Ganguly and A. Paul, Phys. Chem. Chem. Phys., 2016, 18, $25308-25314$

99. S. Sun, B. Mignolet, L. Fan, W. Li, R. D. Levine and F. Remacle, f. Phys. Chem. A, 2017, 121, 1442-1447.

100. R. Shepard, H. Lischka, P. G. Szalay, T. Kovar and M. Ernzerhof, f. Chem. Phys., 1992, 96, 2085-2098.

101. K. Andersson, P.-Å. Malmqvist, B. O. Roos, A. J. Sadlej and K. Wolinski, f. Phys. Chem., 1990, 94, 5483-5488.

102. M. Barbatti, J. J. Szymczak, A. J. A. Aquino, D. Nachtigallová and H. Lischka, f. Chem. Phys., 2011, 134, 014304.

103. M. Barbatti, Z. Lan, R. Crespo-Otero, J. J. Szymczak, H. Lischka and W. Thiel, f. Chem. Phys., 2012, 137, 22A503.

104. F. Peccati, S. Mai and L. González, Phil. Trans. R. Soc. A, 2017, 375, 20160202.

105. A. C. Borin, S. Mai, P. Marquetand and L. González, Phys. Chem. Chem. Phys., 2017, 19, 5888-5894. 
106. B. Sellner, M. Barbatti, T. Müller, W. Domcke and H. Lischka, Mol. Phys., 2013, 111, 2439-2450.

107. M. Ruckenbauer, M. Barbatti, T. Müller and H. Lischka, F. Phys. Chem. A, 2013, 117, 2790-2799.

108. M. Vazdar, M. Eckert-Maksić, M. Barbatti and H. Lischka, Mol. Phys., 2009, 107, 845-854.

109. H. Tao, B. G. Levine and T. J. Martínez, J. Phys. Chem. A, 2009, 113, 13656-13662.

110. J. W. Park and T. Shiozaki, f. Chem. Theory Comput., 2017, 13, 3676-3683.

111. J. P. Zobel, J. J. Nogueira and L. González, Chem. Sci., 2017, 8, 1482-1499.

112. A. Dreuw and M. Head-Gordon, Chem. Rev., 2005, 105, 4009-4037.

113. D. Tuna, D. Lefrancois, Ł. Wolański, S. Gozem, I. Schapiro, T. Andruniów, A. Dreuw and M. Olivucci, f. Chem. Theory Comput., 2015, 11, 5758-5781.

114. I. Tavernelli, B. F. Curchod and U. Rothlisberger, Chem. Phys., 2011, 391, $101-109$.

115. R. Crespo-Otero and M. Barbatti, f. Chem. Phys., 2011, 134, 164305.

116. K. Bhattacharyya and A. Datta, Chem. Eur. f., 2017, 23, 11494-11498.

117. R. Crespo-Otero, M. Barbatti, H. Yu, N. L. Evans and S. Ullrich, ChemPhysChem, 2011, 12, 3365-3375.

118. D. Tuna, N. Došlić, M. Mališ, A. L. Sobolewski and W. Domcke, f. Phys. Chem. B, 2015, 119, 2112-2124.

119. M. Barbatti and R. Crespo-Otero, in Surface Hopping Dynamics with DFT Excited States, ed. N. Ferré, M. Filatov and M. Huix-Rotllant, Springer International Publishing, Cham, 2016, pp. 415-444.

120. A. B. Trofimov and J. Schirmer, 7. Phys. B: At., Mol. Opt. Phys., 1995, 28, 2299.

121. C. Hättig, Adv. Quantum Chem., 2005, 50, 37-60.

122. F. Plasser, R. Crespo-Otero, M. Pederzoli, J. Pittner, H. Lischka and M. Barbatti, f. Chem. Theory Comput., 2014, 10, 1395-1405.

123. A. Prlj, B. F. E. Curchod and C. Corminboeuf, Phys. Chem. Chem. Phys., 2015, 17, 1471914730.

124. S. Mai, F. Plasser, M. Pabst, F. Neese, A. Köhn and L. González, F. Chem. Phys., 2017, 147, 184109.

125. A. I. Krylov, Annu. Rev. Phys. Chem., 2008, 59, 433-62.

126. D. Lefrancois, D. Tuna, T. J. Martínez and A. Dreuw, F. Chem. Theory Comput., 2017, 0, null.

127. B. H. Lengsfield, III, P. Saxe and D. R. Yarkony, F. Chem. Phys., 1984, 81, 4549-4553.

128. I. Fdez. Galván, M. G. Delcey, T. B. Pedersen, F. Aquilante and R. Lindh, F. Chem. Theory Comput., 2016, 12, 3636-3653.

129. H. Lischka, M. Dallos, P. G. Szalay, D. R. Yarkony and R. Shepard, F. Chem. Phys., 2004, 120, 7322-7329.

130. I. Tavernelli, E. Tapavicza and U. Rothlisberger, f. Chem. Phys., 2009, 130, 10.

131. F. Plasser, M. Ruckenbauer, S. Mai, M. Oppel, P. Marquetand and L. González, f. Chem. Theory Comput., 2016, 12, 1207. 
132. E. Tapavicza, I. Tavernelli and U. Rothlisberger, Phys. Rev. Lett., 2007, 98, 023001.

133. J. Pittner, H. Lischka and M. Barbatti, Chem. Phys., 2009, 356, 147 - 152.

134. P.-Å. Malmqvist and B. O. Roos, Chem. Phys. Lett., 1989, 155, $189-194$.

135. J. Finley, P.-A.. Malmqvist, B. O. Roos and L. Serrano-Andrés, Chem. Phys. Lett., 1998, 288, 299-306.

136. F. Plasser, G. Granucci, J. Pittner, M. Barbatti, M. Persico and H. Lischka, f. Chem. Phys., 2012, 137, 22A514.

137. B. A. Heß, C. M. Marian, U. Wahlgren and O. Gropen, Chem. Phys. Lett., 1996, 251, 365-371.

138. P.-A. Malmqvist, B. O. Roos and B. Schimmelpfennig, Chem. Phys. Lett., 2002, 357, 230.

139. F. Neese, f. Chem. Phys., 2005, 122, 034107.

140. F. Neese, WIREs Comput. Mol. Sci., 2012, 2, 73-78.

141. A. Berning, M. Schweizer, H. Werner, P. J. Knowles and P. Palmieri, Mol. Phys., 2000, 98, 1823-1833.

142. S. Mai, T. Müller, P. Marquetand, F. Plasser, H. Lischka and L. González, f. Chem. Phys., 2014, 141, 074105.

143. P. Pyykkö, Chem. Rev., 1988, 88, 563-594.

144. E. van Lenthe, J. G. Snijders and E. J. Baerends, f. Chem. Phys., 1996, 105, 6505-6516.

145. T. Nakajima and K. Hirao, Chem. Rev., 2012, 112, 385.

146. J. Almlöf and P. R. Taylor, Int. F. Quant. Chem., 1985, 27, 743-768.

147. M. Pabst and A. Köhn, f. Chem. Phys., 2008, 129, 214101.

148. B. T. Pickup, Chem. Phys., 1977, 19, 193-208.

149. M. Spanner, S. Patchkovskii, C. Zhou, S. Matsika, M. Kotur and T. C. Weinacht, Phys. Rev. A, 2012, 86, 053406.

150. M. Ruckenbauer, S. Mai, P. Marquetand and L. González, f. Chem. Phys., 2016, 144, 074303.

151. J. C. Tully, f. Chem. Phys., 2012, 137, 22 A301.

152. M. Persico, I. Cacelli and A. Ferretti, f. Chem. Phys., 1991, 94, 5508-5523.

153. M. Spanner and S. Patchkovskii, Phys. Rev. A, 2009, 80, 063411.

154. M. Spanner and S. Patchkovskii, Chem. Phys., 2012, in press.

155. S. Gozem, A. O. Gunina, T. Ichino, D. L. Osborn, J. F. Stanton and A. I. Krylov, J. Phys. Chem. Lett., 2015, 6, 4532-4540.

156. R. Mitrić, J. Petersen, M. Wohlgemuth, U. Werner, V. Bonačić-Koutecký, L. Wöste and J. Jortner, f. Phys. Chem. A, 2011, 115, 3755-3765.

157. W. Arbelo-González, R. Crespo-Otero and M. Barbatti, f. Chem. Theory Comput., 2016, 12, 5037-5049.

158. A. S. Petit and J. E. Subotnik, f. Chem. Phys., 2014, 141, 154108.

159. E. T. Nibbering, H. Fidder and E. Pines, Annu. Rev. Phys. Chem., 2005, 56, 337-367.

160. E. J. Heller, Acc. Chem. Res., 1981, 14, 368-375.

161. F. Plasser and H. Lischka, J. Chem. Phys., 2011, 134, 034309. 
162. F. Plasser, B. Thomitzni, S. A. Bäppler, J. Wenzel, D. R. Rehn, M. Wormit and A. Dreuw, $\mathcal{F}$. Comp. Chem., 2015, 36, 1609-1620.

163. R. L. Martin, J. Chem. Phys., 2003, 118, 4775-4777.

164. M. Head-Gordon, A. M. Grana, D. Maurice and C. A. White, f. Chem. Phys., 1995, 99, 14261-14270.

165. F. Plasser, M. Wormit and A. Dreuw, f. Chem. Phys., 2014, 141, 024106.

166. F. Plasser and H. Lischka, f. Chem. Theory Comput., 2012, 8, 2777-2789.

167. S. A. Bäppler, F. Plasser, M. Wormit and A. Dreuw, Phys. Rev. A, 2014, 90, 052521.

168. A. V. Luzanov and O. A. Zhikol, Int. F. Quant. Chem., 2010, 110, 902-924.

169. F. Plasser, A. J. A. Aquino, W. L. Hase and H. Lischka, J. Phys. Chem. A, 2012, 116, 11151-11160.

170. F. Plasser and H. Lischka, Photochem. Photobiol. Sci., 2013, 12, 1440-52.

171. J. J. Nogueira, F. Plasser and L. González, Chem. Sci., 2017, 8, 5682-5691.

172. S. A. Mewes, J.-M. Mewes, A. Dreuw and F. Plasser, Phys. Chem. Chem. Phys., 2016, 18, 2548-2563.

173. F. Plasser and A. Dreuw, f. Phys. Chem. A, 2015, 119, 1023-1036.

174. D. Cremer and J. A. Pople, f. Am. Chem. Soc., 1975, 97, 1354-1358.

175. J. C. A. Boeyens, f. Cryst. Mol. Struct., 1978, 8, 317.

176. M. Barbatti and H. Lischka, f. Am. Chem. Soc., 2008, 130, 6831-6839.

177. N. Kungwan, F. Plasser, A. J. A. J. A. Aquino, M. Barbatti, P. Wolschann and H. Lischka, Phys. Chem. Chem. Phys., 2012, 14, 9016.

178. C. Bressler and M. Chergui, Annu. Rev. Phys. Chem., 2010, 61, 263-282.

179. G. Sciaini and R. J. D. Miller, Rep. Prog. Phys., 2011, 74, 096101.

180. L. Kurtz, A. Hofmann and R. de Vivie-Riedle, f. Chem. Phys., 2001, 114, 6151-6159.

181. F. Plasser, M. Barbatti, A. J. A. Aquino and H. Lischka, J. Phys. Chem. A, 2009, 113, 8490-8499.

182. C. Chudoba, E. Riedle, M. Pfeiffer and T. Elsaesser, Chemical Physics Letters, 1996, 263, $622-628$.

183. K. Stock, C. Schriever, S. Lochbrunner and E. Riedle, Chemical Physics, 2008, 349, 197 203.

184. C. E. Crespo-Hernández, B. Cohen, P. M. Hare and B. Kohler, Chem. Rev., 2004, 104, 1977-2020.

185. P. M. Hare, C. E. Crespo-Hernández and B. Kohler, Proc. Natl. Am. Soc., 2007, 104, 435-440.

186. C. T. Middleton, K. de La Harpe, C. Su, Y. K. Law, C. E. Crespo-Hernández and B. Kohler, Ann. Rev. Phys. Chem., 2009, 60, 217-239.

187. M. de Vries, Photoinduced Phenomena in Nucleic Acids I, Springer Berlin Heidelberg, 2015, vol. 355, pp. 33-56.

188. H. Kang, K. T. Lee, B. Jung, Y. J. Ko and S. K. Kim, J. Am. Chem. Soc., 2002, 124, 1295812959. 
189. S. Ullrich, T. Schultz, M. Z. Zgierski and A. Stolow, Phys. Chem. Chem. Phys., 2004, 6, 2796-2801.

190. C. Canuel, M. Mons, F. Piuzzi, B. Tardivel, I. Dimicoli and M. Elhanine, f. Chem. Phys., 2005, 122, 074316.

191. J.-W. Ho, H.-C. Yen, W.-K. Chou, C.-N. Weng, L.-H. Cheng, H.-Q. Shi, S.-H. Lai and P.-Y. Cheng, f. Phys. Chem. A, 2011, 115, 8406-8418.

192. G. Bazsó, G. Tarczay, G. Fogarasi and P. G. Szalay, Phys. Chem. Chem. Phys., 2011, 13, 6799-6807.

193. P. Marquetand, J. J. Nogueira, S. Mai, F. Plasser and L. González, Molecules, 2017, 22, 49.

194. R. S. Burkey and C. D. Cantrell, f. Opt. Soc. Am. B, 1984, 1, 169-175.

195. C. Marante, L. Argenti and F. Martín, Phys. Rev. A, 2014, 90, 012506.

196. R. K. Nesbet, Phys. Rev. A, 1976, 14, 1065-1081.

197. V. Tagliamonti, P. Sándor, A. Zhao, T. Rozgonyi, P. Marquetand and T. Weinacht, Phys. Rev. A, 2016, 93, 051401.

198. P. Sándor, V. Tagliamonti, A. Zhao, T. Rozgonyi, M. Ruckenbauer, P. Marquetand and T. Weinacht, Phys. Rev. Lett., 2016, 116, 063002.

199. W. D. M. Lunden, P. Sándor, T. C. Weinacht and T. Rozgonyi, Phys. Rev. A, 2014, 89, 053403. 


\section{List of Figures}

1 State representations and their potentials. In the presence of SOC, the states can be defined by (a) their electronic character (i.e., spectroscopic properties remain mainly unchanged with coordinate), (b) sorting the eigenenergies within one multiplicity (eigenstates of the $\mathrm{MCH}$ ), or (c) diagonalizing the Hamiltonian containing SOCs and then strictly ordering according to energy. In the presence of dipole moment-field couplings (instead of or in addition to SOC) a similar notation is possible (d,e,f). Note that in this case, time is plotted on the $\mathrm{x}$-axis and an electric field with Gaussian envelope leads to time-dependent potentials in the diagonal picture (f). Terms used in the literature for each representation are listed in the panels. . . . . . . . . . . .

2 Comparison of (a) QD and (b) SH simulations. Initially, the wave packet is located on the left on the upper PES, but after a few fs it reaches the nonadiabatic interaction region, where most of the wave packet switches to the lower PES and finally can be found on the right. Smaller parts of the wave packet instead move to the left or remain on the upper PES. In the $\mathrm{SH}$ simulation, the splitting of the wave packet at the interaction region is faithfully reproduced by the swarm of trajectories. . . . . . . . . . . . .

3 Experimental techniques for probing ultrafast nuclear dynamics in molecules: (a) time-resolved photoionization spectroscopy, (b) transient absorption spectroscopy, and (c) time-resolved infrared spectroscopy. FC stands for FranckCondon. . . . . . . . . . . . . . . . . . . . .

4 Simulated population dynamics ${ }^{89}$ (left panels) and photo-ionization yields ${ }^{15}$ (right panels) of (a,c) keto cytosine and (b,d) enol cytosine. The respective signal heights of the photo-ionization yields reflects the ratio of approximately 35:65 for keto:enol cytosine in the gas phase. . . . . . . . . . . .

5 Time-resolved photo-electron yield for gas-phase cytosine computed with SHARC and Dyson norms compared to experimental results from Ref. 189. Adapted from Ref. 15. . . . . . . . . . . . . . . . . . . . . . . . . . . . . 
with spin-orbit coupling:

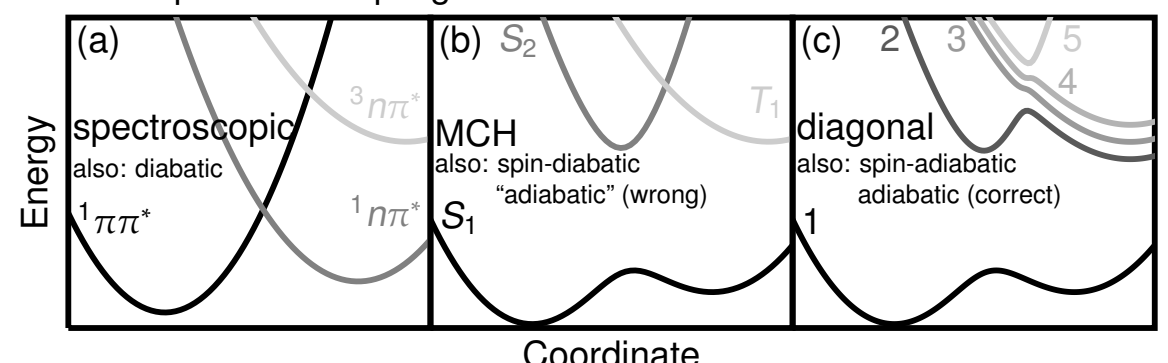

with dipole moment - field coupling:

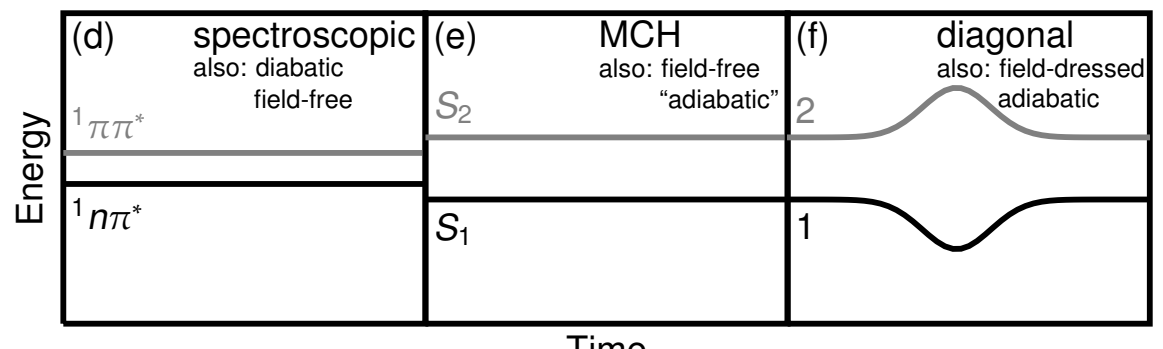

Time

Figure 1: State representations and their potentials. In the presence of SOC, the states can be defined by (a) their electronic character (i.e., spectroscopic properties remain mainly unchanged with coordinate), (b) sorting the eigenenergies within one multiplicity (eigenstates of the $\mathrm{MCH}$ ), or (c) diagonalizing the Hamiltonian containing SOCs and then strictly ordering according to energy. In the presence of dipole moment-field couplings (instead of or in addition to SOC) a similar notation is possible (d,e,f). Note that in this case, time is plotted on the $\mathrm{x}$-axis and an electric field with Gaussian envelope leads to time-dependent potentials in the diagonal picture (f). Terms used in the literature for each representation are listed in the panels. 

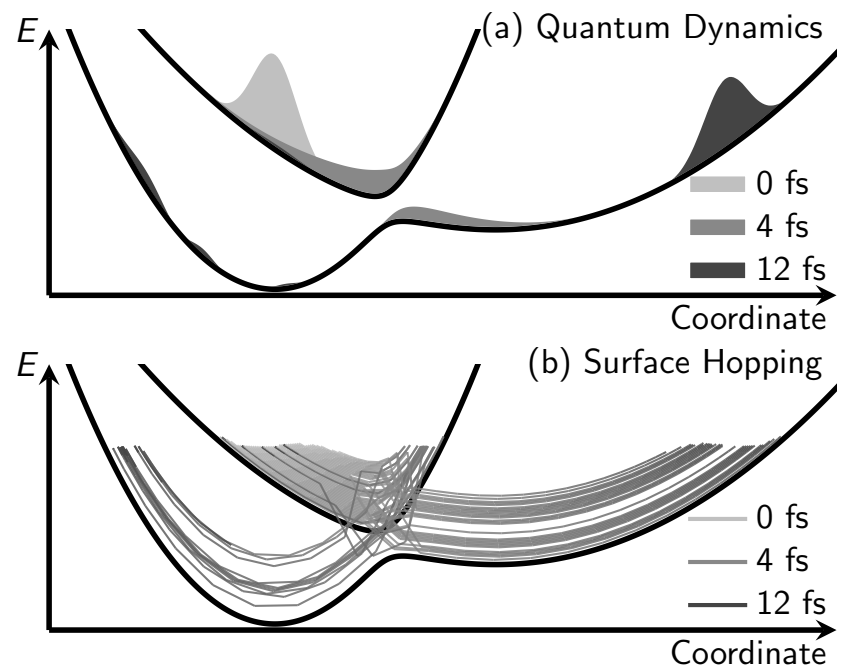

Figure 2: Comparison of (a) QD and (b) SH simulations. Initially, the wave packet is located on the left on the upper PES, but after a few fs it reaches the nonadiabatic interaction region, where most of the wave packet switches to the lower PES and finally can be found on the right. Smaller parts of the wave packet instead move to the left or remain on the upper PES. In the SH simulation, the splitting of the wave packet at the interaction region is faithfully reproduced by the swarm of trajectories. 
(a) TR Photoionization

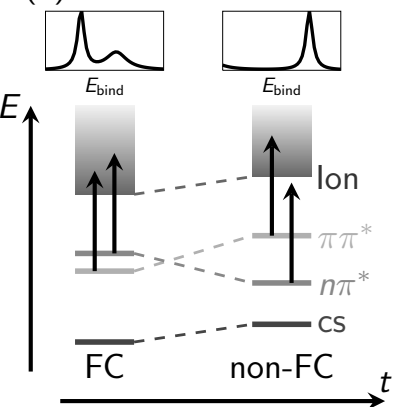

(b) Transient Absorption

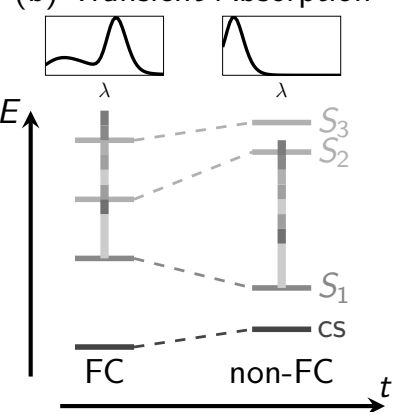

(c) TR Infrared

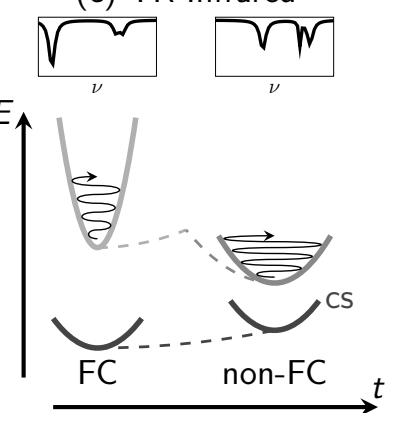

Figure 3: Experimental techniques for probing ultrafast nuclear dynamics in molecules: (a) time-resolved photoionization spectroscopy, (b) transient absorption spectroscopy, and (c) time-resolved infrared spectroscopy. FC stands for Franck-Condon. 


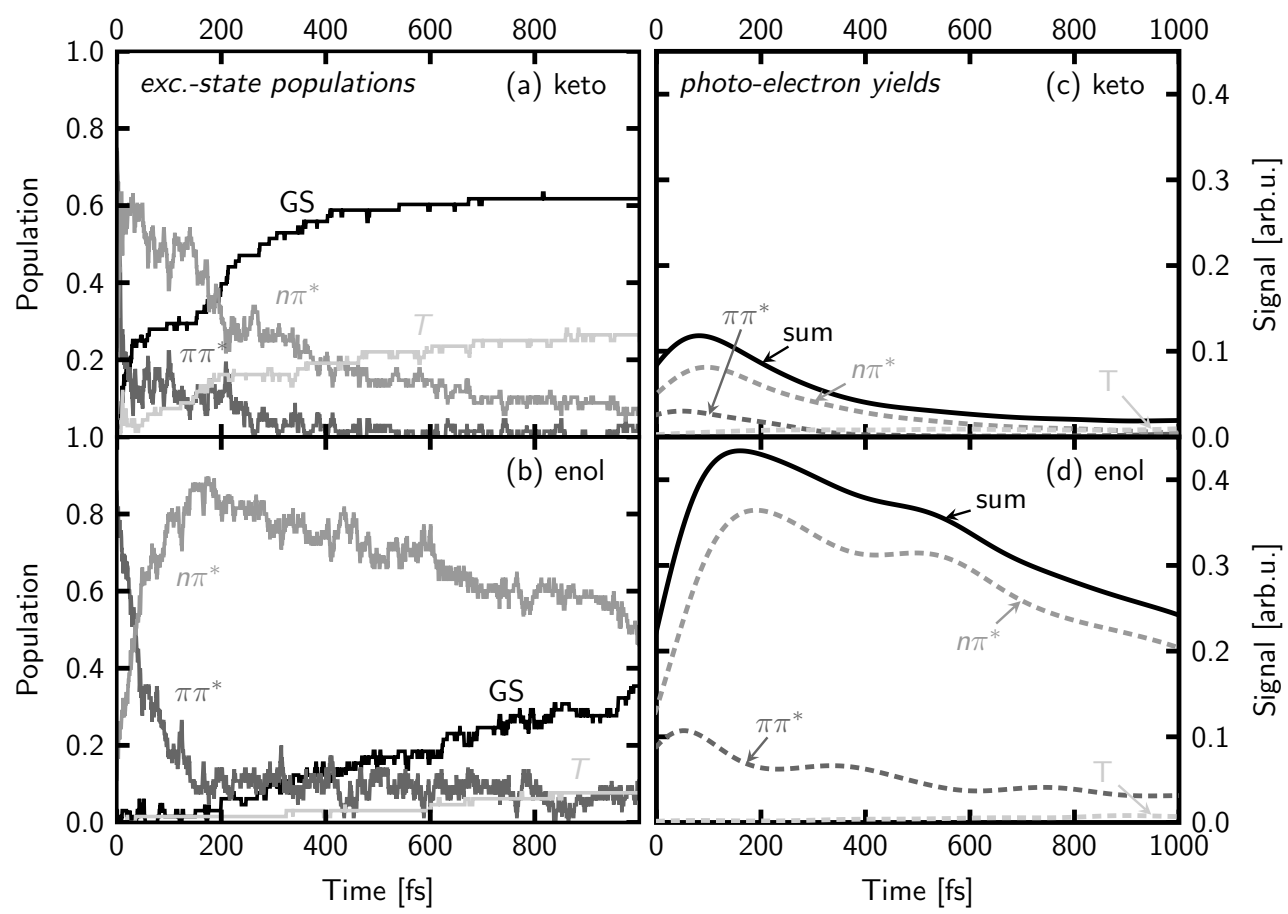

Figure 4: Simulated population dynamics ${ }^{89}$ (left panels) and photo-ionization yields ${ }^{15}$ (right panels) of (a,c) keto cytosine and (b,d) enol cytosine. The respective signal heights of the photo-ionization yields reflects the ratio of approximately 35:65 for keto:enol cytosine in the gas phase. 


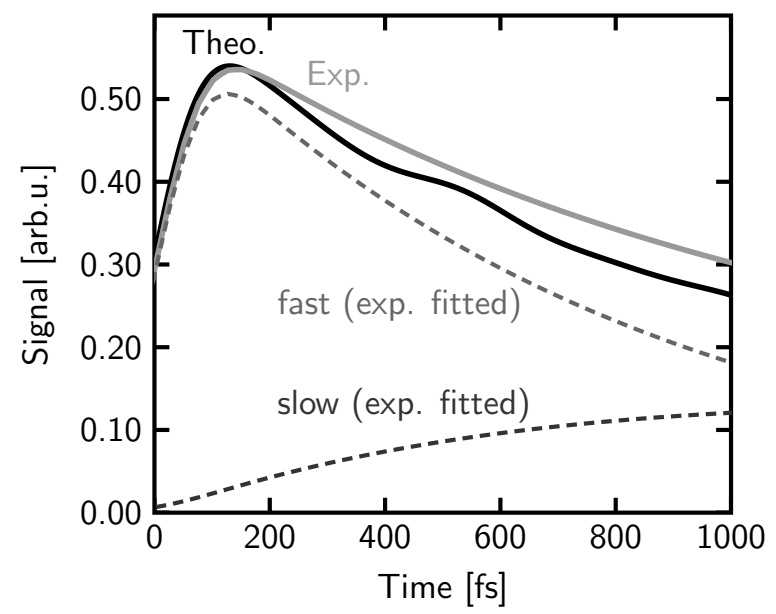

Figure 5: Time-resolved photo-electron yield for gas-phase cytosine computed with SHARC and Dyson norms compared to experimental results from Ref. 189. Adapted from Ref. 15. 


\section{List of Tables}

1 Overview over the quantum chemistry interfaces of SHARC. For each method and program, the table shows which multiplicities $\left(S^{2}\right)$ and which quantities are available.

2 Fitted time constants for the excited-state populations and integrated ionization yields depicted in Fig. 4. Note that the experimentally extracted fit constants correspond to 820 fs and 3200 fs. ${ }^{189}$ 
Table 1: Overview over the quantum chemistry interfaces of SHARC. For each method and program, the table shows which multiplicities $\left(S^{2}\right)$ and which quantities are available.

\begin{tabular}{|c|c|c|c|c|c|c|c|c|c|}
\hline Method & Program & $S^{2}$ & Grad. & NAC & $\mathrm{OVL}^{a}$ & SOC & $\mathrm{TDM}^{b}$ & Dyson & Refs. \\
\hline \multirow[t]{3}{*}{$\overline{\text { SA-CASSCF }}$} & MOLPRO & any & $\sqrt{ }$ & $\sqrt{ }$ & $\sqrt{ }$ & $\sqrt{ }$ & $\sqrt{ }$ & $\sqrt{ }$ & $20,22,24,89$ \\
\hline & MOLCAS & any & $\sqrt{ }$ & & $\sqrt{ }$ & $\sqrt{ }$ & $\sqrt{ }$ & $\sqrt{ }$ & 29,30 \\
\hline & Columbus & any & $\sqrt{ }$ & $\sqrt{ }^{c}$ & $\sqrt{ }$ & $\sqrt{ }^{c}$ & $\sqrt{ }$ & $\sqrt{ }$ & \\
\hline MR-CISD & Columbus & any & $\sqrt{ }$ & $\sqrt{ }^{c}$ & $\sqrt{ }$ & $\sqrt{ }^{c}$ & $\sqrt{ }$ & $\sqrt{ }$ & $27,28,104,105$ \\
\hline MS-CASPT2 & Molcas & any & $\sqrt{ }^{d}$ & & $\sqrt{ }$ & $\sqrt{ }$ & $\sqrt{ }$ & $\sqrt{ }$ & 26 \\
\hline \multirow[t]{2}{*}{ TD-DFT } & $\mathrm{ADF}$ & any & $\sqrt{ }$ & & $\sqrt{ }$ & $\sqrt{ }{ }^{e}$ & $\sqrt{f}$ & $\sqrt{ }$ & 31 \\
\hline & GAUSSIAN & any & $\sqrt{ }$ & & $\sqrt{ }$ & & $\sqrt{ }^{f}$ & $\sqrt{ }$ & \\
\hline $\mathrm{ADC} 2$ & Turbomole & $\mathrm{S}, \mathrm{T}$ & $\sqrt{ }$ & & $\sqrt{ }$ & $\sqrt{ }$ & $\sqrt{ }$ & & 124 \\
\hline $\mathrm{CC} 2$ & Turbomole & $\mathrm{S}, \mathrm{T}$ & $\sqrt{ }$ & & $\sqrt{ }$ & & $\sqrt{ }^{f}$ & & \\
\hline
\end{tabular}

${ }^{a}$ OVL: wave function overlaps; ${ }^{b}$ TDM: transition dipole moments; ${ }^{c}$ either NAC or SOC, but not both at the same time; ${ }^{d}$ numerical gradients; ${ }^{e}$ SOCs only between singlets and triplets; ${ }^{f}$ TDMs only between $S_{0}$ and excited singlets. 
Table 2: Fitted time constants for the excited-state populations and integrated ionization yields depicted in Fig. 4. Note that the experimentally extracted fit constants correspond to $820 \mathrm{fs}$ and $3200 \mathrm{fs}{ }^{189}$

\begin{tabular}{lcc}
\hline Contribution & $\tau_{\text {exc.-state population }}[\mathrm{fs}]$ & $\tau_{\text {photo-electron yield }}[\mathrm{fs}]$ \\
\hline \multicolumn{3}{c}{- Keto -} \\
$\pi \pi^{*}$ & 25 & 21 \\
$n \pi^{*}$ & 216 & 960 \\
$\mathrm{~T}$ & 745 & 310 \\
\hline \multicolumn{3}{c}{ - Enol -} \\
$\pi \pi^{*}$ & 40 & 57 \\
$n \pi^{*}$ & 1513 & 1080 \\
$\mathrm{~T}$ & 6220 & - \\
\hline
\end{tabular}

